\title{
DECIPHERING SOLAR MAGNETIC ACTIVITY. I. ON THE RELATIONSHIP BETWEEN THE SUNSPOT CYCLE AND THE EVOLUTION OF SMALL MAGNETIC FEATURES
}

\author{
Scott W. McIntosh ${ }^{1}$, Xin Wang $^{1,2}$, Robert J. Leamon $^{3}$, Alisdair R. Davey ${ }^{4}$, Rachel Howe $^{5}$, Larisza D. Krista ${ }^{6}$, \\ anna V. Malanushenko ${ }^{3,7}$, Robert S. Markel ${ }^{1}$, Jonathan W. Cirtain ${ }^{8}$, Joseph B. Gurman $^{9}$, \\ William D. PeSnell ${ }^{9}$, AND Michael J. ThOMPSON ${ }^{1}$ \\ ${ }^{1}$ High Altitude Observatory, National Center for Atmospheric Research, P.O. Box 3000, Boulder, CO 80307, USA; mscott@ucar.edu \\ ${ }^{2}$ School of Earth and Space Sciences, Peking University, Beijing 100871, China \\ ${ }^{3}$ Department of Physics, Montana State University, Bozeman, MT 59717, USA \\ ${ }^{4}$ Harvard-Smithsonian Center for Astrophysics, 60 Garden Street, Cambridge, MA 02138, USA \\ ${ }^{5}$ School of Physics and Astronomy, University of Birmingham, Edgbaston, Birmingham, B15 2TT, UK \\ ${ }^{6}$ Cooperative Institute for Research in Environmental Sciences, University of Colorado, Boulder, CO 80205, USA \\ ${ }^{7}$ Lockheed-Martin Solar and Astrophysics Laboratory, 3251 Hanover Street, Org. A021S, Bldg. 252, Palo Alto, CA 94304, USA \\ ${ }^{8}$ Marshall Space Flight Center, Code ZP13, Huntsville, AL 35812, USA \\ ${ }^{9}$ Solar Physics Laboratory, NASA Goddard Space Flight Center, Greenbelt, MD 20771, USA \\ Received 2014 April 2; accepted 2014 July 2; published 2014 August 8
}

\begin{abstract}
Sunspots are a canonical marker of the Sun's internal magnetic field which flips polarity every $\sim 22 \mathrm{yr}$. The principal variation of sunspots, an $\sim 11 \mathrm{yr}$ variation, modulates the amount of the magnetic field that pierces the solar surface and drives significant variations in our star's radiative, particulate, and eruptive output over that period. This paper presents observations from the Solar and Heliospheric Observatory and Solar Dynamics Observatory indicating that the $11 \mathrm{yr}$ sunspot variation is intrinsically tied to the spatio-temporal overlap of the activity bands belonging to the $22 \mathrm{yr}$ magnetic activity cycle. Using a systematic analysis of ubiquitous coronal brightpoints and the magnetic scale on which they appear to form, we show that the landmarks of sunspot cycle 23 can be explained by considering the evolution and interaction of the overlapping activity bands of the longer-scale variability.
\end{abstract}

Key words: Sun: activity - Sun: atmosphere - Sun: evolution - Sun: general - Sun: interior - Sun: rotation sunspots

Online-only material: color figures

\section{INTRODUCTION}

Early investigations of the enigmatic spots on the Sun revealed that their number waxes and wanes over a period of about $11 \mathrm{yr}$ (e.g., Schwabe 1844), a phenomenon that became known as the sunspot (or solar) cycle. It was subsequently found that the latitudinal distribution of sunspots, and their progression over their evolutionary cycle, followed a trail from mid-solar latitudes (about $\pm 35^{\circ}$ ) at first appearance, through solar maximum (when their number is at its greatest), to their eventual disappearance near the equator (about $\pm 5^{\circ}$ ) into the relative calm of solar minimum (e.g., Maunder 1904). Following minimum, a couple of years later, the spots appear again at mid-latitudes and their progression to the equator starts afresh, defining the start of the next sunspot cycle.

The pattern that sunspot locations make in this cyclical progression when latitude is plotted versus time is dubbed the "butterfly diagram" and has become an iconic image of the Sun's variability. Continuing this rapid pace of discovery, G. E. Hale and colleagues subsequently determined that sunspots were locations of intense magnetic field (Hale et al. 1919) and that in consecutive butterfly wings (sunspots in the same hemisphere but belonging to the next cycle), the sunspots had opposite magnetic polarities (Hale 1924). Indeed, they had discovered that the sign of the prevalent magnetic field in each hemisphere of the Sun undergoes a complete period every 22 yr (e.g., Babcock 1959; Harvey 1992).

The radiative and particulate output of the Sun is strongly modulated by the $11 \mathrm{yr}$ sunspot cycle. The continued observation and cataloging of sunspots since the pioneering observations of
Schwabe, Maunder, and Hale have provided us with the most striking proxy of the Sun's activity cycle, which induces both violent (short-term: "Space Weather") and gradual (long-term: "Space Climate") changes in the Sun-Earth connection. The ever-increasing reliance of humanity on space-based technology has reached the point where understanding the origin and impact of the magnetic activity of our star is imperative.

In the following sections, we present an analysis of small ubiquitous features in the Sun's photosphere and corona which, when identified in images and tracked over time, illustrate a systematic magnetic evolution over a considerably longer interval than the $11 \mathrm{yr}$ waxing and waning of sunspots. Furthermore, the observed temporal progression appears to illustrate the latitudinal variation of oppositely signed toroidal magnetic flux systems, or "bands," belonging to Hale's 22 yr magnetic polarity cycle. This observational finding confirms the observational work of Wilson et al. (1988) and Harvey (1992), who were the first to identify the observational traces of an "extended solar cycle" (ESC; see also Tappin \& Altrock 2013). Finally, we expand on our analysis, and that of these pioneering papers, to show that the landmarks of sunspot cycle 23 can be phenomenologically described by considering the latitudinal interaction between these overlapping longer-lived bands.

\section{OBSERVATIONS AND ANALYSIS}

A map of the magnetic range of influence (or "MRoI"; McIntosh et al. 2006) is constructed from a line-of-sight magnetogram in pixel-by-pixel fashion and is defined as the (radial) distance from that pixel at which the total signed flux of 
the enclosed region is zero. The MRoI is a measure of magnetic balance, or the effective lengthscale over which we would expect the overlying corona to be connected or closed.

Figure 1 illustrates the correspondences between Solar Dynamics Observatory (SDO)/Helioseismic and Magnetic Imager (HMI) MRoI and coronal structures. McIntosh et al. (2014) discussed the four apparent lengthscales which are present in a typical MRoI map: a scale at the resolution limit of $S D O / \mathrm{HMI}$ peaking at $\sim 5 \mathrm{Mm}$, which is most likely a signature of magnetic fields organized on granular scales; a very large contribution peaking at $\sim 25 \mathrm{Mm}$ which is consistent with the mean size of quiet Sun supergranules; a distribution of 100-250 Mm scale objects which is consistent with the spatial dimension of giant convective cells (see, e.g., Miesch 2005); and the very long connective lengthscales of active regions and coronal holes for which the method was originally conceived (McIntosh et al. $2006,2007,2010)$. The determination of the 100-250 Mm scale by McIntosh et al. (2014) is strong evidence that a giant convective scale exists and is readily identifiable. Isolation and tracking of features associated with this rotationally driven global convective scale offer insight into the evolution of the magnetic fields that reach to the very bottom of the solar convection zone. McIntosh et al. (2014) also expanded on the correspondence first noted by McIntosh (2007) that EUV brightpoints (BPs; e.g., Vaiana et al. 1973) tend to form recurrently in the vicinity of concentrations of MRoI on the 100-250 Mm scale. McIntosh et al. (2014) dubbed these features "g nodes" because of their apparent connection to the giant convective scale (i.e., inferred to be vertices associated with the giant cells of that size).

The circles shown in Figure 1(b) are the locations of the EUV BPs detected in the SDO Atmospheric Imaging Assembly (AIA; Lemen et al. 2012) $193 \AA$ imaging channel. Details of the BP detection and tracking algorithms are available in the literature (see, e.g., McIntosh \& Gurman 2005; Hara \& Nakakubo-Morimoto 2003), although we have taken steps to improve the reliability of the detections in subsequent years. Following image calibration and cosmic-ray removal (using mission-provided software), we construct a background image $\left(I_{b}\right)$ using a $40 \mathrm{Mm} \times 40 \mathrm{Mm}$ smooth version of that image $(I)$. BPs are defined as spatially small (2-20 Mm) $3 \sigma$ enhancements of the original image over the background image. That is, we construct a " $\sigma$ " image $\left(\left(I-I_{b}\right) / \sqrt{I_{b}}\right)$. A $\sigma$ image constructed in this way can account for subtle differences from instrument to instrument, e.g., from Solar and Heliospheric Observatory $(\mathrm{SOHO}) /$ EUV Imaging Telescope (EIT) and SDO/AIA, and provides significantly more robust BP determination $(\mathrm{S}$. W. McIntosh et al. 2014, in preparation). From the resulting histogram of $\sigma$ image values, we use one, two, and three standard deviations above the mean value to identify the thresholds for BP detection-with the $3 \sigma$ detections being the most reliable. After defining the BP detection thresholds, we isolate contiguous pixel groups in the images, computing their center position and radius of gyration. Only $3 \sigma$ regions with radii between 2 and $20 \mathrm{Mm}$ are defined as belonging to BPs, and in the case presented we make no effort to separate the quiet and active region BPs. The use of $3 \sigma$ BPs (as in this paper) allows for a large degree of confidence in the detection and latitudinal variation of the BPs. Panels (a) and (b) of Figure 1 show an SDO/AIA $193 \AA$ image of the solar corona (1) without and (2) with the BPs identified, respectively. The same detection and identification methodology can be applied to the full-disk MRoI maps (panel (d)) to identify only the spatial concentration of the $100-250 \mathrm{Mm}$ scale, i.e., the g nodes.
We repeat the $\mathrm{BP}$ and g-node identification process for the SOHO/EIT (Delaboudinière et al. 1995), SDO/AIA, SOHO/ Michelson Doppler Imager (MDI; Scherrer et al. 1995), and SDO/HMI (Scherrer et al. 2012) image and magnetogram archives and the results are shown in Figure 2 as latitude-time plots sampling only the variation $\pm 5^{\circ}$ from the central meridian. These measures are compensated for the variation in the inclination of the Sun's poles relative to the Sun-Earth line (note the sinusoidal nature of the highest-latitude regions). Panel (a) shows the latitude-time variation of the EUV BP density in the SOHO/EIT $195 \AA$ and SDO/AIA BP $193 \AA$ images (the interested reader is pointed to the figures of McIntosh \& Gurman 2005, for reference). We note a paucity of BPs detected in this fashion above $\pm 55^{\circ}$ latitude and the appearance of multiple "bands" of BPs in the 1996-1998 and 2006-2011 time frames. The latitudinal variation of the (central meridian) MRoI is shown in panel (b) where perhaps the most striking pattern is the clear delineation of evolution above and below $55^{\circ}$ latitude. Below $55^{\circ}$ latitude, the MRoI pattern evolves toward the equator-like the BPs-but the region above does not. Within the limits of solar $B$-angle visibility (and projection effects of line-of-sight magnetic fields), the behavior of MRoI at high latitudes is roughly constant over the solar cycle with little migration. The only excursions occur near solar maximum with a preponderance of magnetic flux elements that cancel over short lengthscale. This change in MRoI behavior starts in 1999 in both hemispheres, but the dominance of short lengthscales in the northern hemisphere appears to end earlier ( 2002) than in the southern hemisphere ( 2003). Panel (c) shows the complex latitude-time evolution of the g-node density. We note that the dark patches in the g-node density distribution are artifacts of the MRoI technique, in that it is often difficult to see scales of the order of 100-250 Mm inside active regions and coronal holes which have spatially distributed regions of large MRoI (see panel (b) and Figure 1(d)). Like the BPs, there is a significant reduction in the number of $\mathrm{g}$ nodes above $55^{\circ}$ latitude and there also appear to be low-density, asymmetric bands of g nodes that reach up to $55^{\circ}$ at apparently different times, approximately 2002 in the north and 2004 in the south. Furthermore, at the same time as the multiple overlapping bands of BPs (1996-1998 and 2006-2011), there are large concentrations of $g$ nodes in the region below $55^{\circ}$.

\subsection{Identifying and Fitting Activity Bands}

To quantify the discussion of Figure 2, we use the daily histograms of BP and g-node density to identify and track the latitudinal migration of these features. We use the following recipe to map the positions of the activity bands in the latitude-time figures.

1. Averaging the latitudinal BP and g-node distributions over a running 28 day window, we construct the histograms of each as a function of latitude like those shown in Figure 3 where we draw comparison with those published previously (e.g., Golub \& Vaiana 1978).

2. We find the latitudinal locations of the histogram peaks, allowing for a maximum of four (two per hemisphere). The histogram peaks are fitted using Gaussians from which we can determine their mean latitude and width with the latter being employed as an (upper) estimate of the error in establishing the mean latitude. The fits are manually verified, and the peak locations are sorted by latitude and 

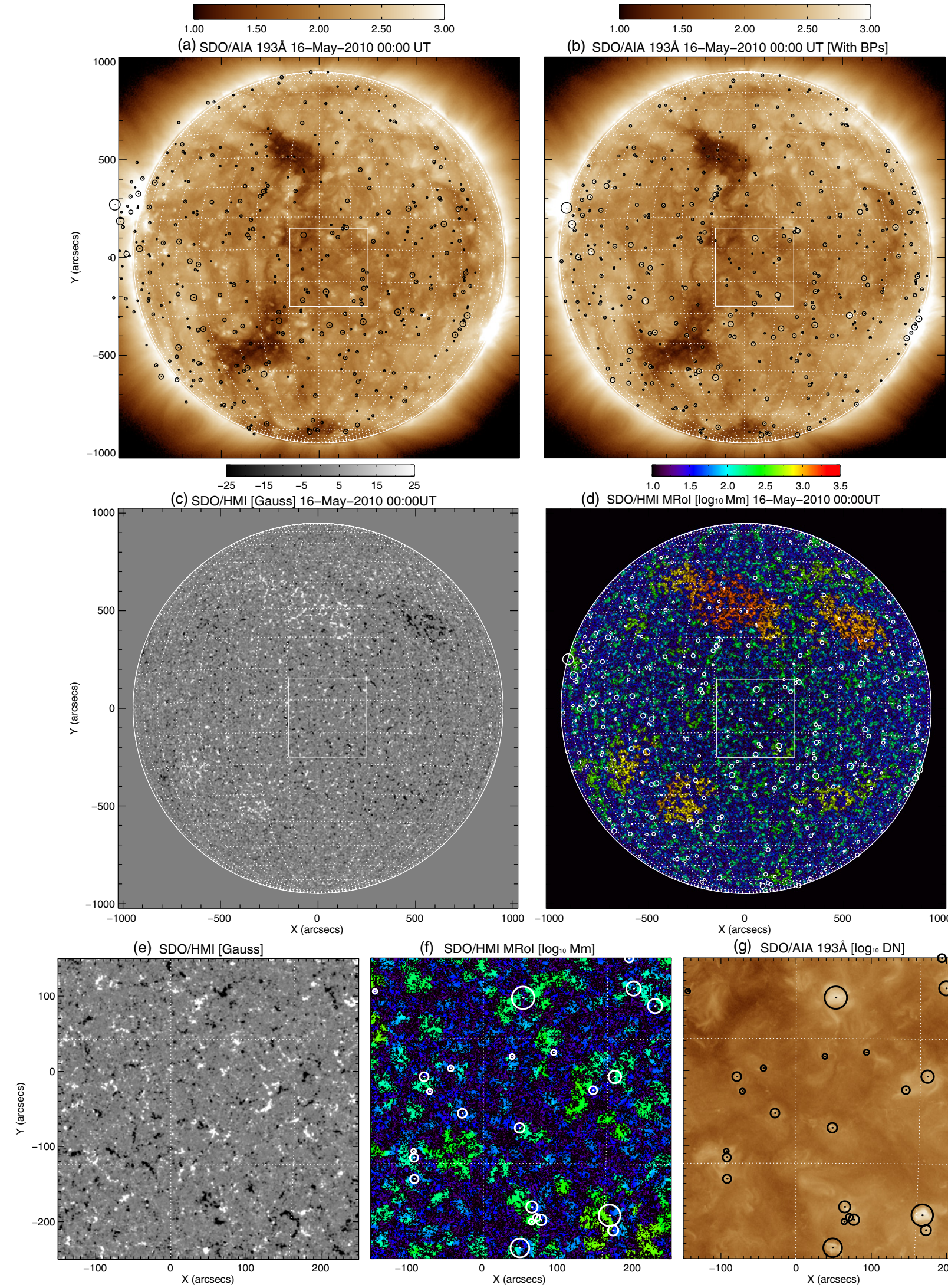

(d) SDO/HMI MRol $\left[\log _{10} \mathrm{Mm}\right]$ 16-May-2010 00:00UT
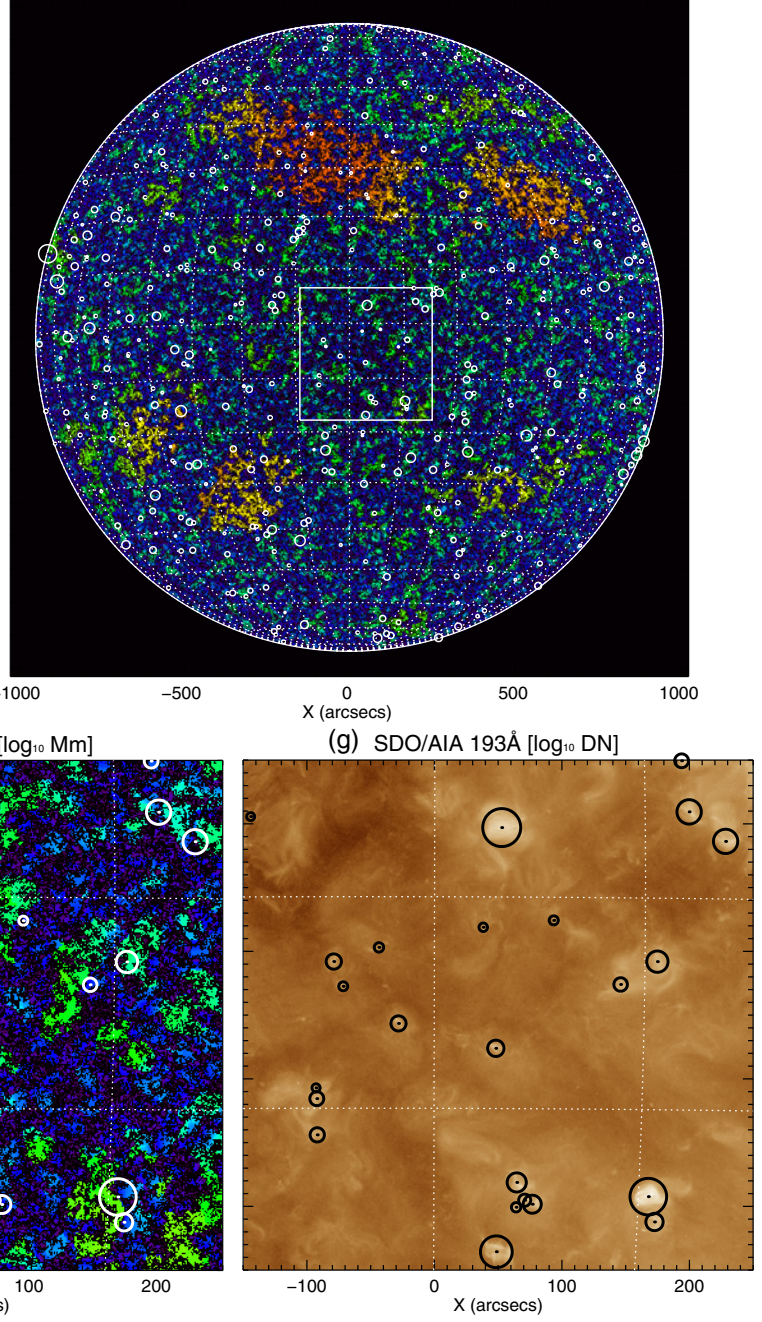

Figure 1. Adapted from McIntosh et al. (2014). EUV brightpoints (BPs) and surface magnetism. Full-disk SDO/AIA image of the solar corona in the $193 \AA$ A channel (a). Panel (b) shows the locations of the detected coronal BPs (black circles). Panel (c) shows the corresponding full-disk SDO/HMI line-of-sight magnetogram, while panel (d) shows the derived "magnetic range of influence" (MRoI) map. The MRoI map also has the coronal BP locations marked (white circles). We compare the inset regions of panels (a) through (d) (white squares), the SDO/HMI magnetogram (e), the MRoI (f), and the coronal environment in SDO/AIA $193 \AA$ (g). McIntosh et al. (2014) noted a strong spatial correspondence between the BP location and the concentrations of MRoI which display a 100-250 Mm connective lengthscale.

(A color version of this figure is available in the online journal.) 
(a) Merged SOHO/EIT 195Å and SDO/AIA 193Å EUV Brightpoints
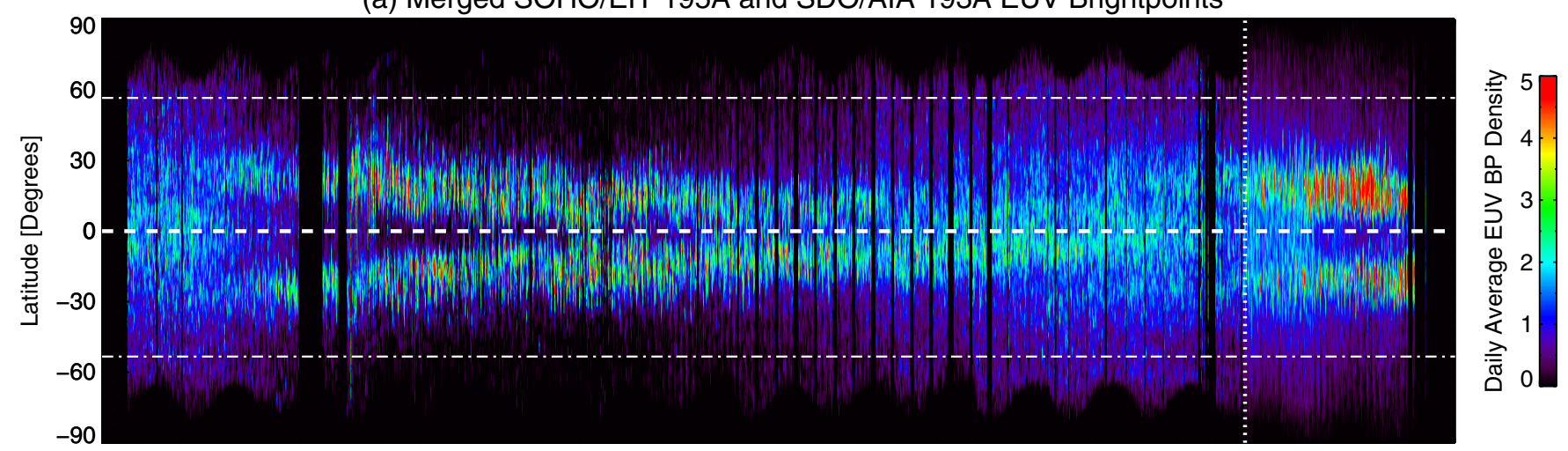

(b) Merged SOHO/MDI and SDO/HMI MRol

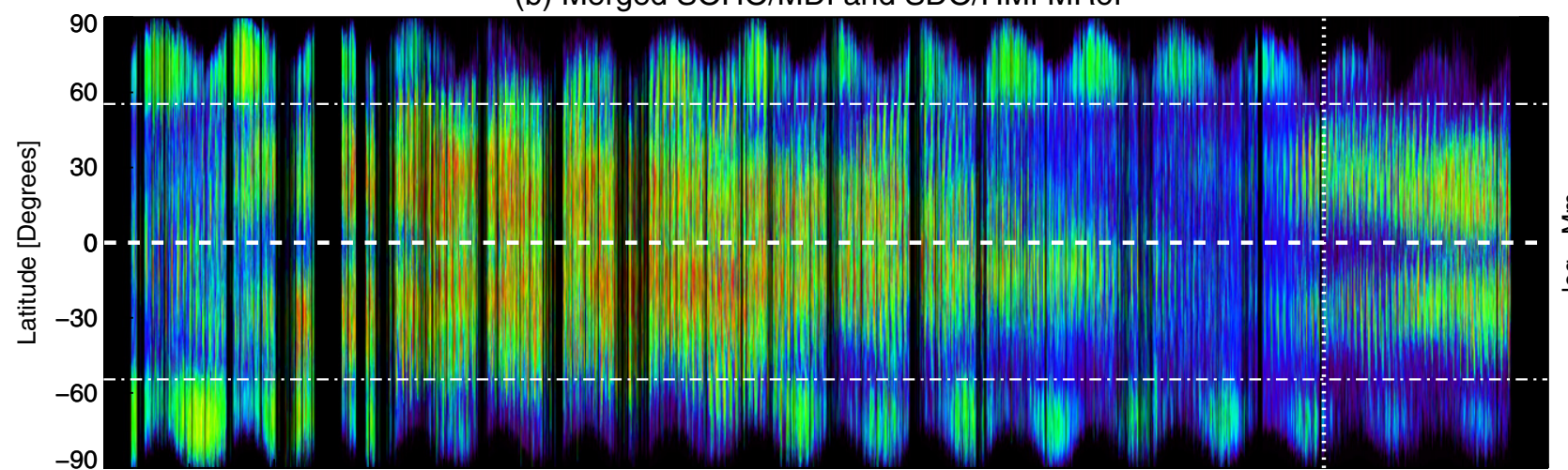

(c) Merged SOHO/MDI and SDO/HMI 100-250Mm MRol g-Nodes

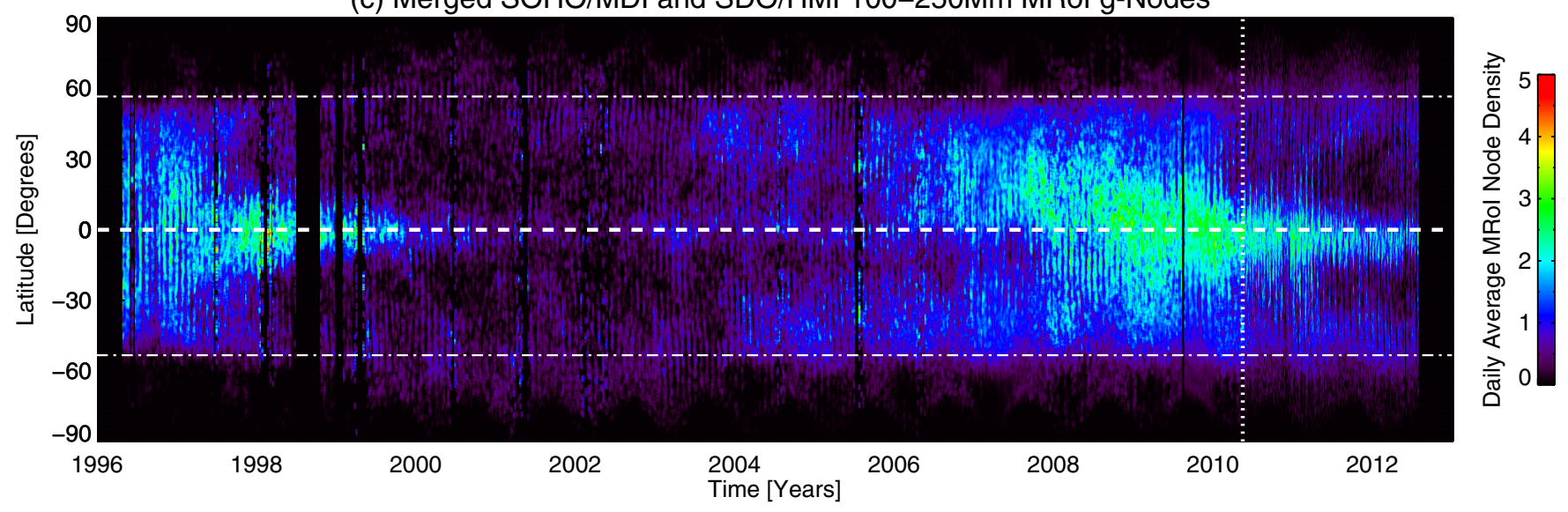

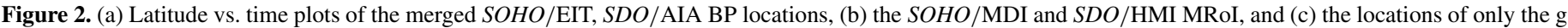

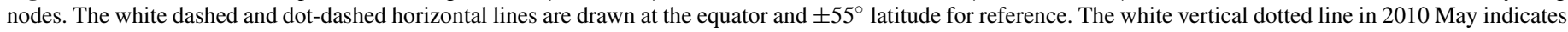
the transition from $\mathrm{SOHO}$ to $\mathrm{SDO}$ diagnostics.

(A color version of this figure is available in the online journal.)

associated with a particular activity band for the next step. Figure 3 shows example fits for g-node and BP distributions at two distinct time steps, noting that in the $7 \mathrm{yr}$ separating the samples, these bands have traveled from $\sim 42^{\circ}$ to $\sim 20^{\circ}$ in each hemisphere. As the bands migrate closer to the equator, it becomes more difficult to isolate them and will associate the same peak and width with one band in each hemisphere.

3. After repeating step 2 for all times, we use the peak locations and widths associated with each band to map their latitudinal progression. A straight line is chosen to fit these points for each band under the assumption that it is the functional form that is minimally consistent with the data points. The results of this analysis are shown in Figure 4.
From our analysis of Figure 4, it appears that the migrating bands of g node and BPs, which we will refer to hereafter as "activity bands," appear to meet, or terminate, at the equator. The three different sets of bands can be associated with solar cycles 22 (green), 23 (red), and 24 (blue). For the currently visible portion of the solar cycle 24 activity bands, the fitted migration gradients are consistent with each other $\left(3.05 \pm 0.11 \mathrm{yr}^{-1}\right.$ in the northern hemisphere and $3.16 \pm 0.09^{\circ} \mathrm{yr}^{-1}$ in the south) within the uncertainty of the fit. These migratory rates are higher by about $1^{\circ} \mathrm{yr}^{-1}$ than the values for the portions of the cycle 22 (green bars) and cycle 23 (red bars) activity bands shown. This indicates that the (linear) approximation used to describe the activity band motion is not the most accurate and that the bands may regularly slow down as they approach the equator, 


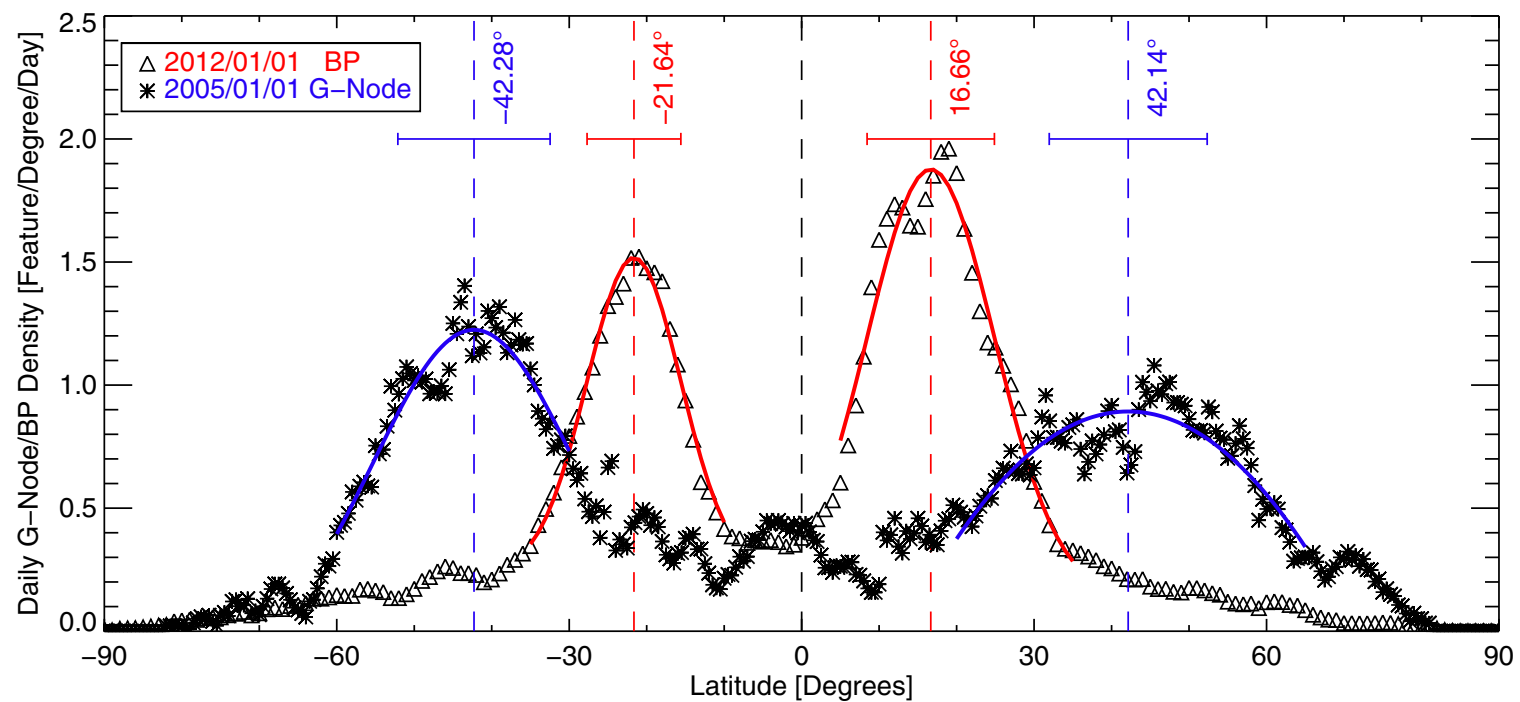

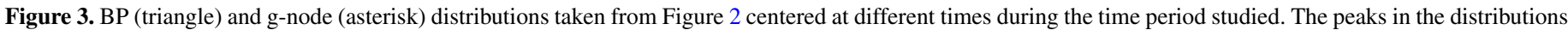

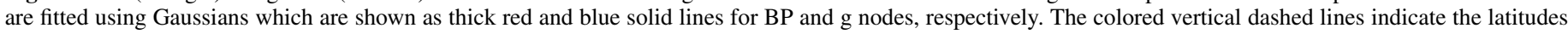
of the relevant distribution peaks while the horizontal bars show the Gaussian $\sigma$ for the distribution fit.

(A color version of this figure is available in the online journal.)

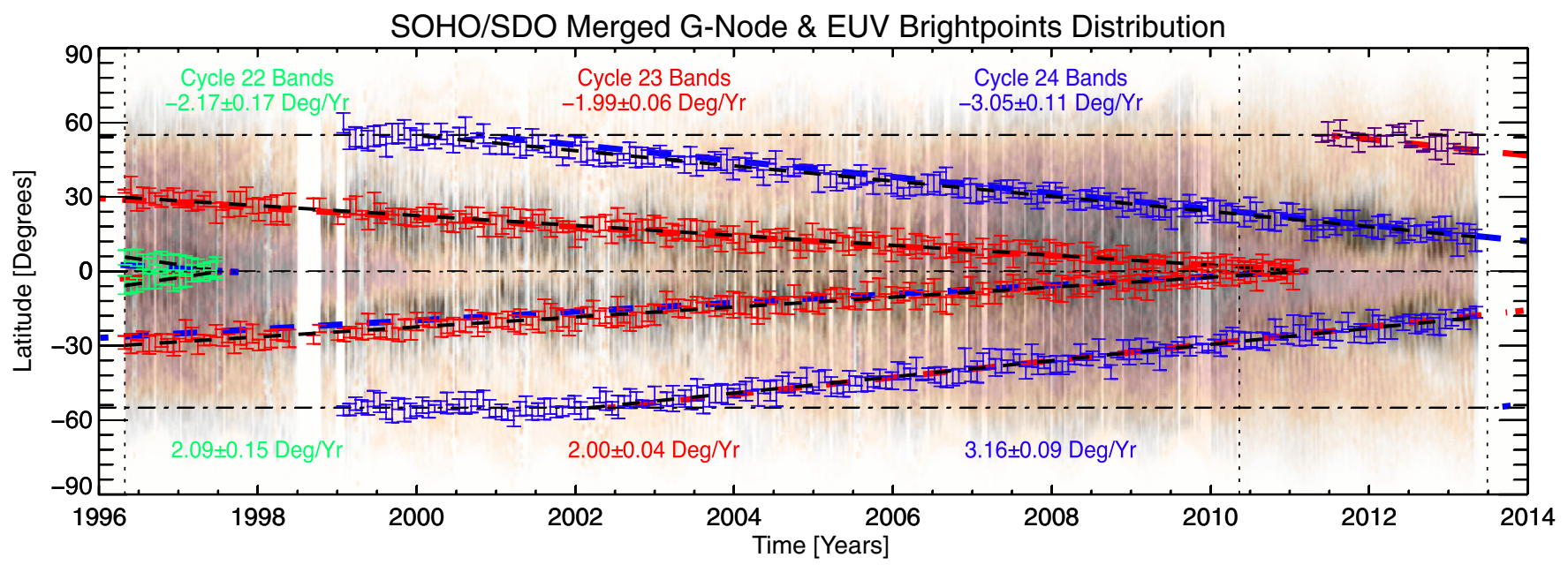

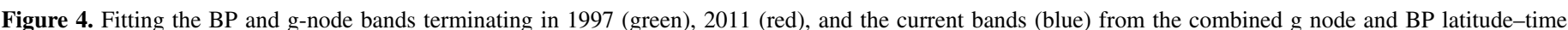

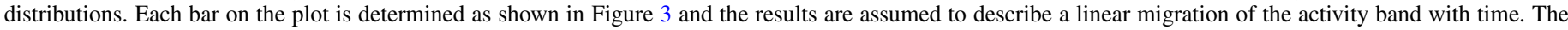

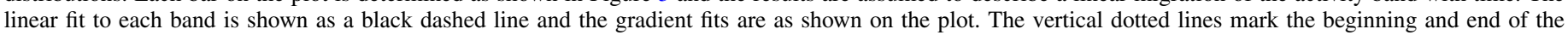
observation sample.

(A color version of this figure is available in the online journal.)

but only time will tell. Note that the purple points, occurring at high latitudes in the northern hemisphere in late 2011, indicate the possible visibility and migratory start of the cycle 25 activity band. If these bands do connect to the sunspot formation bands, then they last significantly longer that the $\sim 11 \mathrm{yr}$ for which sunspots are visible in each cycle (see below) and would appear to slow down as the cycles come to an end.

To illustrate, and validate, the apparent termination points, Figure 5 shows the $\mathrm{SOHO} / \mathrm{EIT}$ and $\mathrm{SDO} / \mathrm{AIA} \mathrm{BP}$ distributions in 1996-1999 (panel (a)) and 2010-2013 (panel (b)). In each case, we see that the latitudinal distribution of BPs following this point in time-1997 August and 2011 March, respectively, - are starkly different.

\subsection{The Space-Time Progression of Small-scale Features}

Figure 6 extends the analysis of BPs, g nodes, and the MRoI over the $17 \mathrm{yr}$ record of synoptic coronal and photospheric observations accrued by $\mathrm{SOHO} / \mathrm{EIT}$, $\mathrm{SOHO} / \mathrm{MDI}, \mathrm{SDO} / \mathrm{AIA}$, and $S D O / \mathrm{HMI}$ (see Figure 2). Panels (a)-(c) repeat those shown in Figure 2, however, we now place the activity bands inferred from the g-node and BP density histograms (Figure 4) on top of the latitude-time plots. For further comparison, we show the hemispherically averaged residual to solar differential rotation inferred from MDI and HMI Dopplergrams at a depth of $0.993 R_{\odot}$ in panel (d) (Howe 2009). This reveals a pattern known as the "torsional oscillation" (e.g., Hathaway 2010; Labonte \& Howard 1982; Charbonneau et al. 1999b), or a measure that has been interpreted as a map of the largescale zonal flows in the solar interior (e.g., Hathaway 1996; Ulrich 2010).

Again, now assisted by the linear fits to the activity bands developed in Section 2.1, the latitudinal progression toward the equator with time is clearly visible in all quantities plotted in the panels of the figure. We immediately note the (general) cor- 
(a) SOHO/EIT 195Å EUV Brightpoints

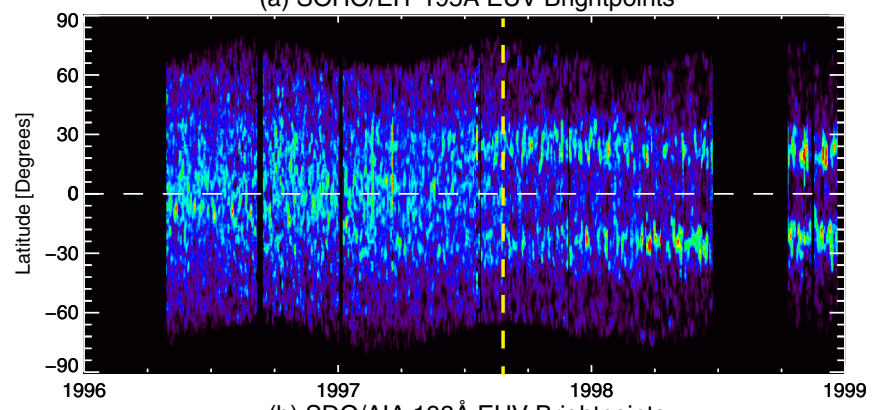

(b) SDO/AIA 193Å EUV Brightpoints

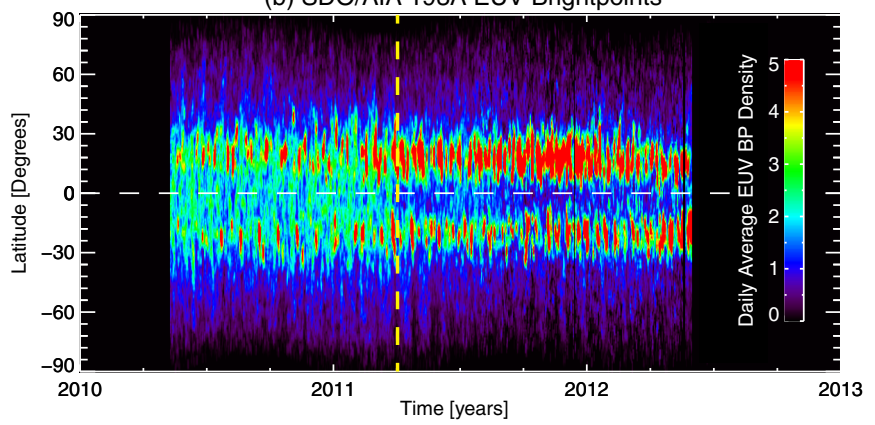

Figure 5. Latitude-time BP distributions for (a) SOHO/EIT $195 \AA$ through the solar cycle 22/23 minimum (1997-1999) and (b) the solar cycle 23/24 minimum (2010-2012) for SDO/AIA $193 \AA$. In these panels, we see bands coming to an end in 1997 August and 2011 March, respectively. The latitudinal variation in BPs shows a marked change following termination at the times marked with vertical dashed yellow lines.

(A color version of this figure is available in the online journal.)

respondence between the surface-magnetism-inferred activity bands and the torsional oscillation but, due to the hemispheric averaging of the latter, some departures are visible. The equatorward migration of BPs and g nodes, and their link to the torsional oscillation, provides further evidence of the deep rooting of these magnetic field concentrations (McIntosh et al. 2014); otherwise, one would expect their motion to be poleward with the flux elements caught in the poleward meridional circulation (e.g., Sheeley 2005; Charbonneau 2010). We see again that that the dot-dashed lines at $\pm 55^{\circ}$ latitude divide the poleward and equatorward behavior, something that is especially clear in panel (d), where we see the latitude at which the torsional oscillation pattern diverging with one pattern going poleward and another going equatorward. The importance and relevance of $\pm 55^{\circ}$ will be discussed in a later section.

We see that there is strong hemispheric asymmetry in the magnetic activity of solar cycle 23 (as is the focus of McIntosh et al. 2013). In the present case, we see that the small-scale magnetic activity bands start their equatorward migration from high latitudes asymmetrically. After appearing in 1999 (seen as horizontally extended clusters of g nodes and in the torsional oscillation at high latitude), the northern band started migrating from $55^{\circ}$ in 2000 while the southern band started migrating later-some time in 2002. It is unclear why the bands, although appearing at the same time, would start migrating equatorward with such a significant offset, but the responsible physical mechanism is likely the root cause of the current hemispheric asymmetry in activity (McIntosh et al. 2013). With the aid of the activity band linear fits (Figure 4), we can establish that there are relatively short periods of time when there is only one migrating activity band visible in each hemisphere. Much of the time, the system in each hemisphere appears to have overlapping bands, as we have noted earlier, with the overlap most easily visible in the BP latitudinal distributions between 1996 and 1998, and 2006 and 2011, i.e., during the solar cycle 22/23 and 23/24 solar minima (e.g., McIntosh et al. 2013). Hereafter, we will refer to the combination of hemispheric activity bands across the solar equator as a "chevron."

The combined data sample presented herein spans only $17 \mathrm{yr}$. The activity bands visible in the latitude-time plots must exist considerably longer than the $\sim 11 \mathrm{yr}$ sunspot cycle and we deduce that they are more closely related to the $\sim 22$ yr magnetic activity cycle. For example, the high-latitude chevron (starting in 2000) has progressed to a latitude of $20^{\circ}$ in approximately $13 \mathrm{yr}$, a latitude reached by the lower-latitude chevron in 2002, approximately $9 \mathrm{yr}$ before it disappeared. The present analysis of BP and g-node evolution in solar cycle 23 (and the start of cycle 24 ) is strengthened by considering the pioneering observational work of Wilson et al. (1988) and Harvey (1992), who studied BPs, "ephemeral active regions," and bright calcium faculae over solar cycles 19 through 21 with Wilson et al. (1988) including the torsional oscillation for cycle 21 . Our analysis both reproduces and confirms the earlier analyses to the degree that, when combined, we have directly observed a systematic progression of small-scale magnetic flux from high to low latitudes that spans close to five sunspot cycles.

\section{COMPARING ACTIVITY BAND PROGRESSION WITH THE HEMISPHERIC SUNSPOT NUMBER}

To go beyond the analysis of Wilson et al. (1988) (and Harvey 1992), we now explore the relationship between the activity bands and the modulation of the sunspot cycle by placing them in context with the variation in the (monthly) hemispheric sunspot number (hSSN) and the (http://solarscience.msfc.nasa.gov/greenwch.shtml) United States Air Force (USAF) sunspot archive in Figure 7. The thick gray, red, and blue vertical dashed lines in the figure are landmarks of sunspot cycle 23. The gray lines mark the times at which the low-latitude chevrons appear to terminate at the equator (in 1997 August and 2011 April; Figure 5).

For the two visible terminations, we see that the sunspots of the upcoming cycle appear rapidly with great abundance and increasing strength for the remaining activity band in each hemisphere after the termination. Incidentally, this transition happens as the higher-latitude band passes $\sim 30^{\circ}$ latitude. From these examples, we infer that the gray lines define the start of the ascending phase of sunspot cycles 23 and 24. The thick dashed red and blue vertical lines mark the asymmetric times when the high-latitude $\left( \pm 55^{\circ}\right)$ bands start their progression to the equator in the northern and southern hemispheres. We see that the start of these lines coincides in time with the hemispheric sunspot maxima, and hence mark the start of the declining phase in each hemisphere.

The thin red (2006 January) and blue (2007 September) dotted lines mark when the higher-latitude activity bands pass $45^{\circ}$ - this starts a period of time when all four activity bands begin to overlap at low latitudes. At these times, the sunspots in each hemisphere rapidly begin to wane in size and number. This period of maximum overlap coincides with the activity/sunspot minimum between solar cycles 23 and 24, a state which appears to persist until the next gray dashed line when the low-latitude bands terminate.

Figure 8 illustrates our interpretation of the cycle 23 activity bands in terms of the underlying magnetism and its variation with time. At the start of the $\mathrm{SOHO}$ era (the activity minimum 
(a) Merged SOHO/EIT 195Å and SDO/AIA 193Å EUV Brightpoints
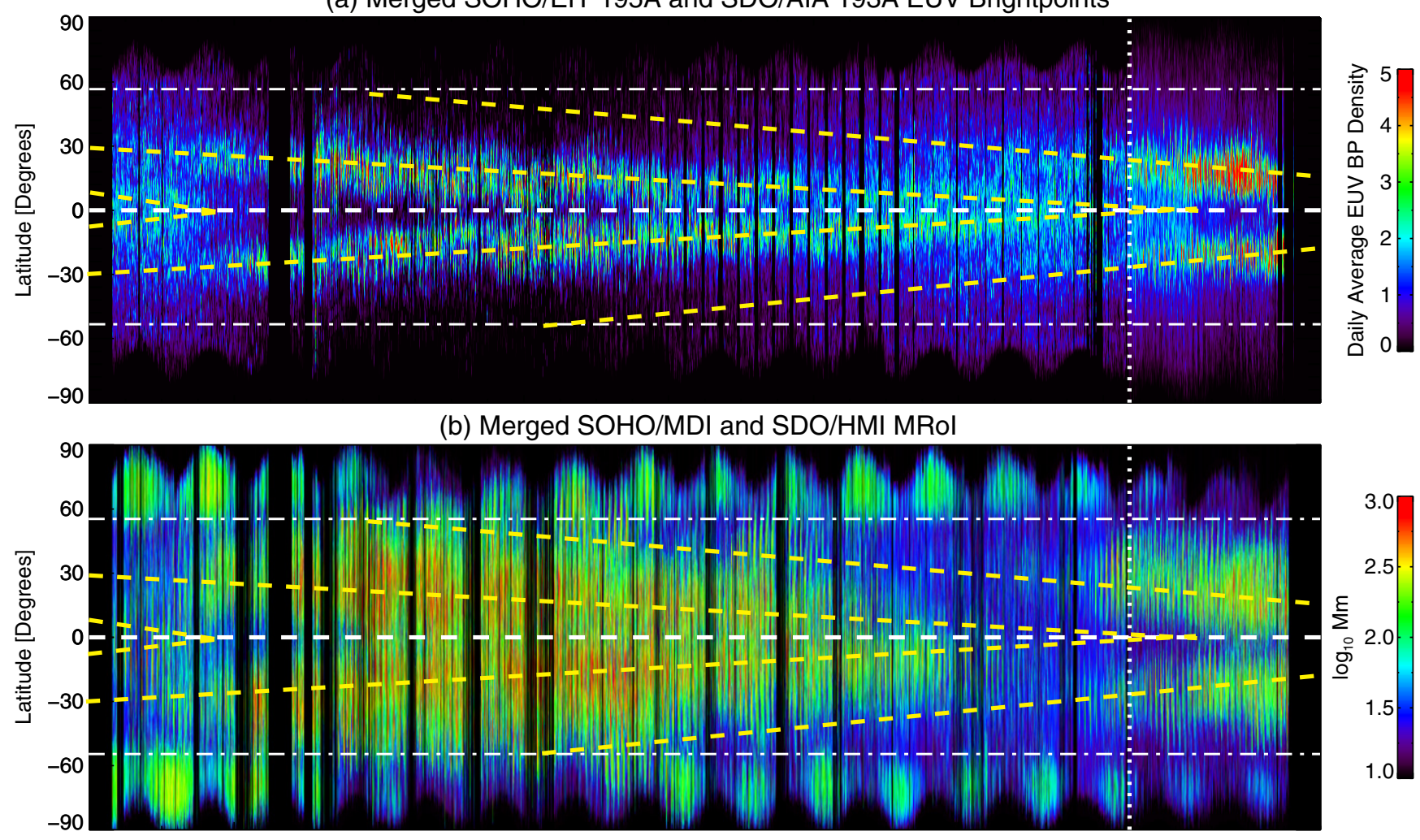

(c) Merged SOHO/MDI and SDO/HMI 100-250Mm MRol Nodes
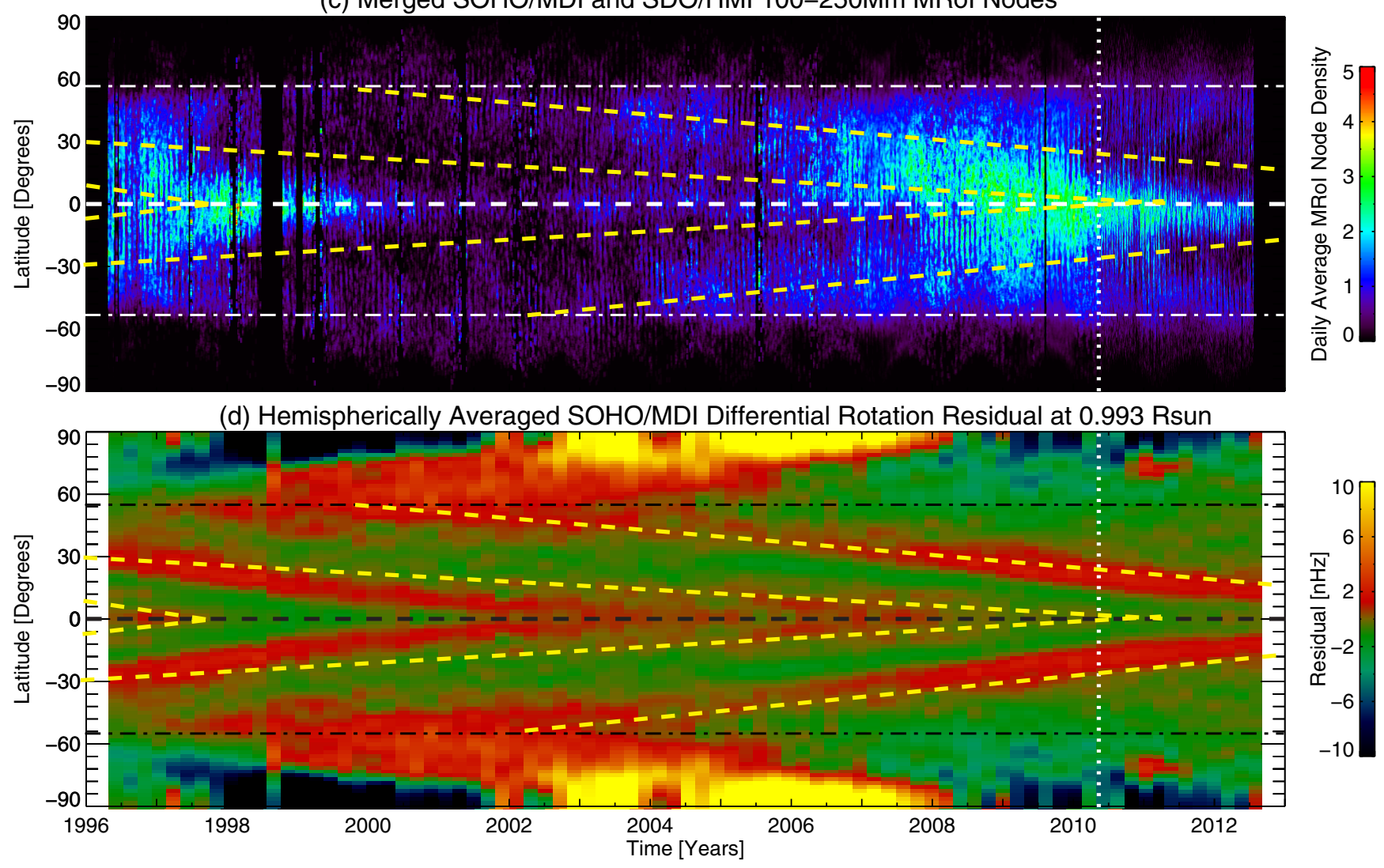

Figure 6. (a) Latitude-time plots of the merged $S O H O$ /EIT, SDO/AIA BP density distribution, (b) the $S O H O / \mathrm{MDI}$ and $S D O / \mathrm{HMI}$ MRoI, (c) the g-nodes density distribution, and (d) the (hemispherically symmetrized) torsional oscillation near the solar surface (Howe et al. 2000). The dot-dashed horizontal lines are drawn at $\pm 55^{\circ}$ latitude and separate the polar and equatorial regions. The yellow dashed bands are drawn using a combination of panels (a) and (c) (as explained in the text and illustrated in Figure 4), using only the pieces that migrate in latitude.

(A color version of this figure is available in the online journal.) 
(a) ROB/SIDC Sunspot Number

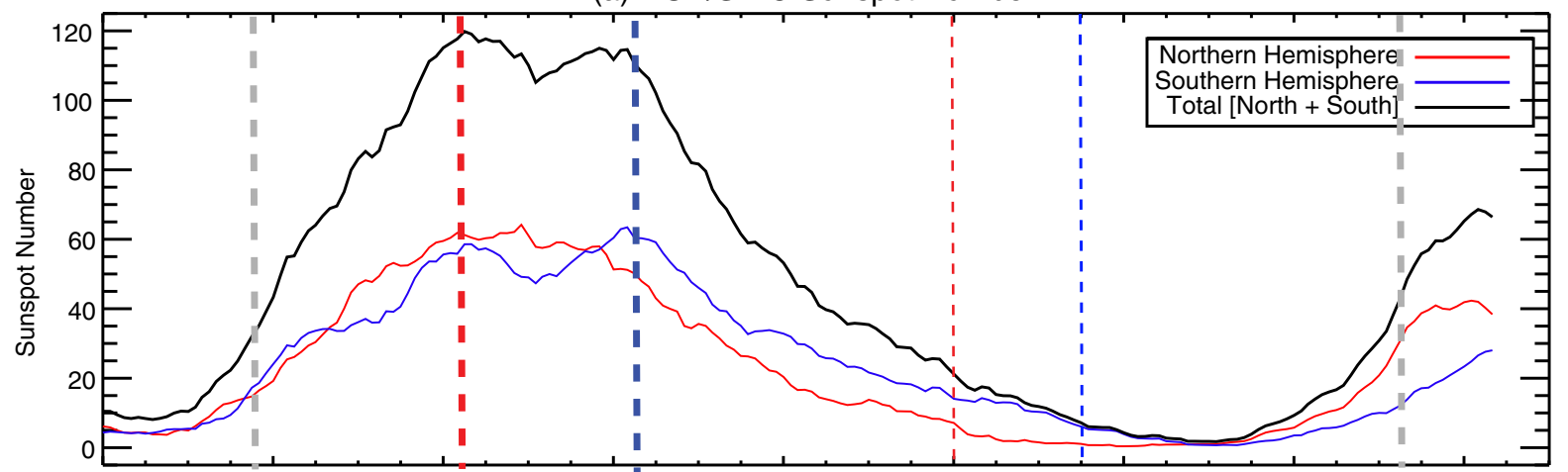

(b) USAF Sunspot Area By Hemisphere
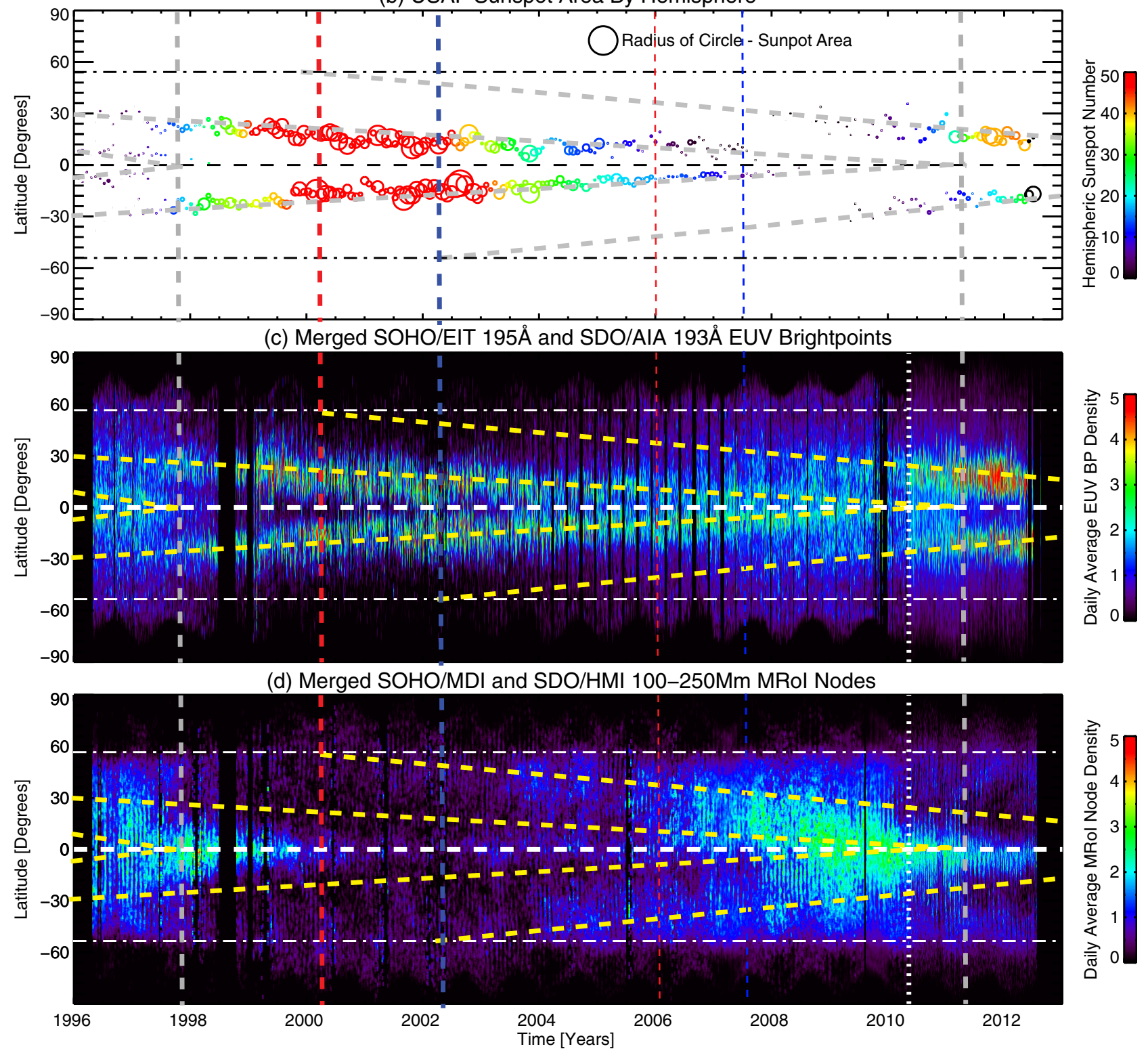

Figure 7. Highlight of the phases of solar cycle 23 and the initial phase of solar cycle 24. Panel (a) shows the smoothed Royal Observatory of Brussels Solar Influences Data Center total SSN and the SSN decomposed by hemisphere (hSSN; north: red; south: blue). Panel (b) shows the latitudinal variation of the SSN and sunspot areas from the United States Air Force Sunspot area archive. The circles drawn are color-coded by the hSSN and their radii reflect the area of the solar disk (in millionths) covered by sunspots averaged over a 28 day period. Panels (c) and (d) are reproductions of the BP and g-node latitude-time plots in Figure 6 and the chevrons in dashed yellow lines are also taken from Figure 6. The red, blue, and gray dashed vertical lines mark the hemispheric sunspot maxima in the north and south, and the apparent activity band termination points (see text and Figure 5). The white vertical dotted lines indicate the transition from SOHO to SDO diagnostics in 2010 May. (A color version of this figure is available in the online journal.) 


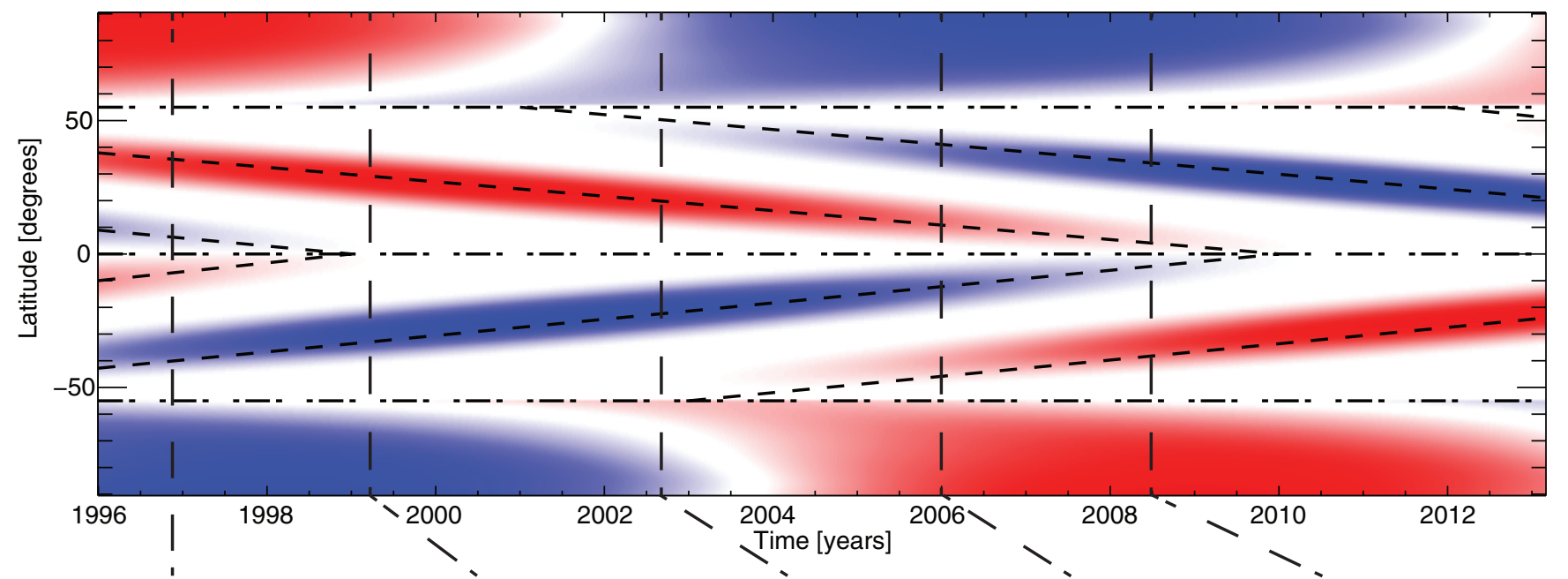

(a) $22 / 23$ Solar Minimum

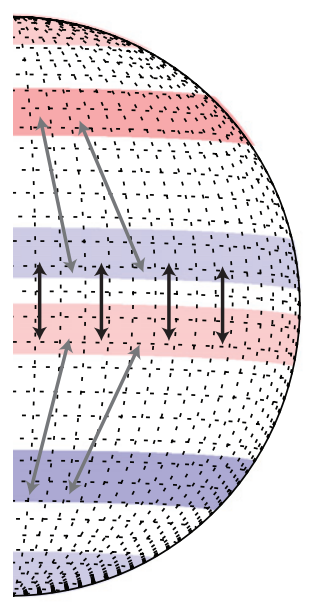

(b) 23 Ascending Phase

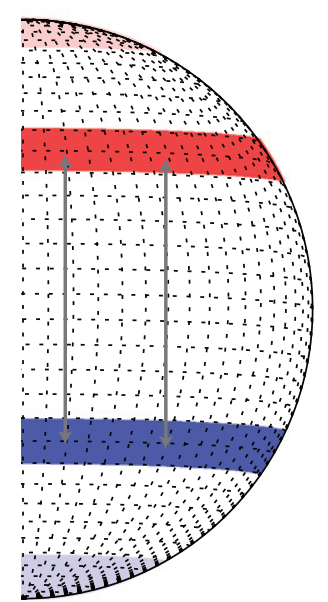

(c) 23 Solar Max Declining Phase Onset

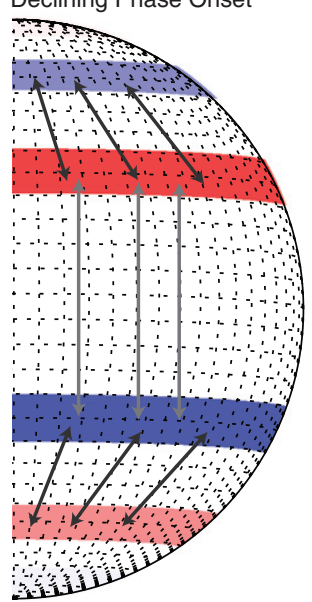

(d) 23 Declining Phase

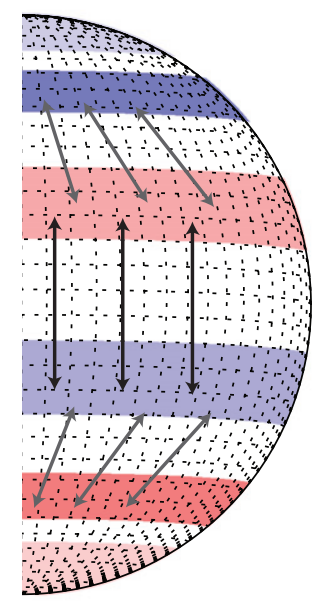

(e) $23 / 24$ Minimum

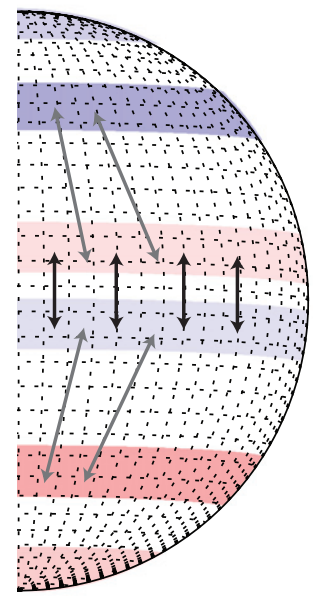

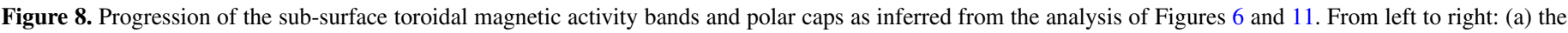

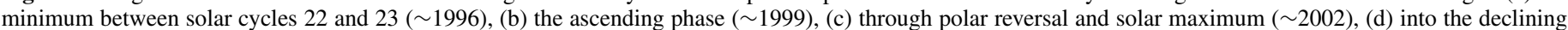

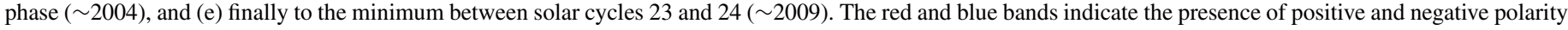

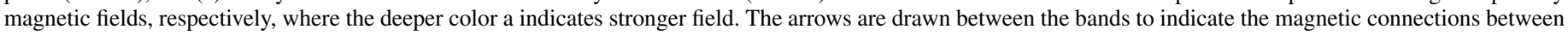
the bands in their hemisphere and across the equator.

(A color version of this figure is available in the online journal.)

of 1996/1997), the net signed flux of the four bands of opposite sign cancel each other within their own hemisphere and across the equator. The termination of the low-latitude bands in 1997 leaves only a single activity band in each hemisphere. The cancelation of the oppositely signed bands across the equator significantly increases the flux density of the remaining highlatitude band in each hemisphere, which then permits the rapid formation and buoyant rise of sunspots on those bands. As we approach solar maximum, a new activity band starts to appear at $\sim 55^{\circ}$ latitude. While sitting at that latitude, some of the magnetic flux in that band emerges and is caught in the surface meridional flow and is advected poleward, and eventually cancels the existing polar field above $55^{\circ}$. As the new activity band starts to migrate equatorward, the magnetic flux that it contains begins to interact with that of the lower-latitude (oppositely signed) band in the same hemisphere. The increased interaction reduces the local flux density in the low-latitude bands, reducing their ability to buoyantly produce sunspots. The result will be a net reduction in available flux, and hence activity. In this picture, the sunspot maximum in each hemisphere is the time when this intra-hemispheric interaction starts afresh. As time progresses and the four (alternating-polarity) activity bands migrate toward the equator, the oppositely signed activity bands interact across the equator as well as in the same hemisphere and the situation becomes the equatorial reflection of the previous solar minimum.

Based on the correspondence of these observational data sets, we deduce the following as the landmarks of the solar (sunspot) cycle.

1. "Solar minimum" is the period of time during which the oppositely signed toroidal flux systems in each hemisphere, and across the equator, mutually cancel.

2. The "ascending phase" of the sunspot cycle involves only one activity band per hemisphere and commences with the end of solar minimum, that is, when the trans-equatorial flux systems cancel.

3. "Solar maximum" is the time at which the new high-latitude flux system begins migrating toward the equator.

4. The "descending phase" of the sunspot cycle involves two activity bands per hemisphere. There is likely a complex state of sub-surface interaction between these bands. 


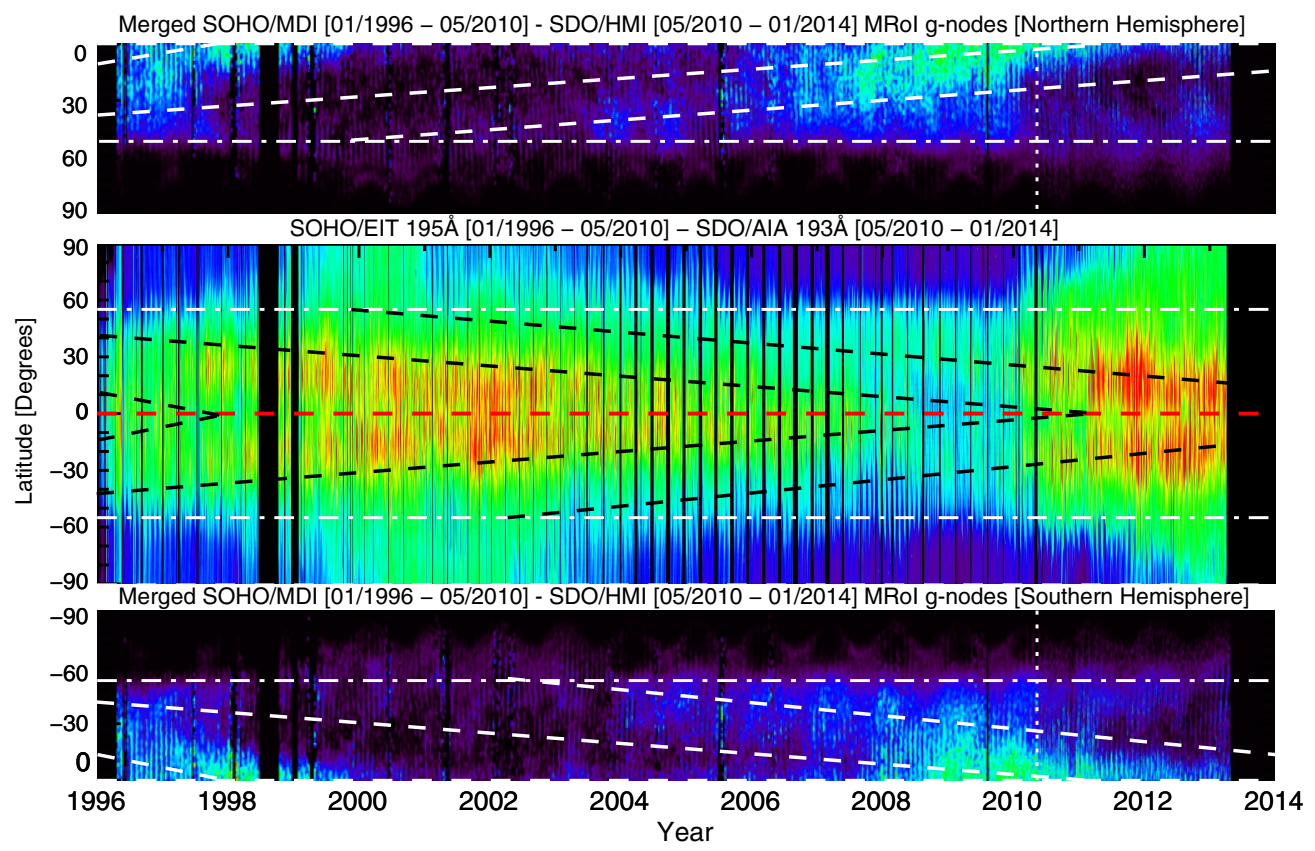

Figure 9. Comparison of the latitudinal progression of coronal emission around the solar limb from SOHO/EIT (195 Å) and SDO/AIA (193 $\AA$ ) with the latitudinal progression of the g nodes in each hemisphere (north-top; south-bottom). The central panel is created by integrating the emission in an annulus of extent $1.15-1.25 R_{\odot}$. The inclined dashed lines illustrating the migratory path of the activity belts are taken from Figure 6 . The horizontal dot-dashed lines mark $\pm 55^{\circ}$ latitude. Note the correspondence in each hemisphere between the duration of the poleward surge and the length of time that the g-node activity band spends at $\pm 55^{\circ}$, and their clear difference in duration.

(A color version of this figure is available in the online journal.)

\section{SUPPORTING EUV OBSERVATIONS OF CYCLE 23 EVOLUTION}

We find further support for the BP and g-node observations of cycle 23 presented above in different analyses of coronal EUV images from $S O H O$ and $S D O$ : the essential nature of $\pm 55^{\circ}$, the length of time spent by the activity bands at $\pm 55^{\circ}$, the short-term temporal variability intrinsic to the system, and the equatorward migration of the activity bands. Figures 9-10 use different analysis techniques to increase the physical richness of the physical picture and the intrinsic coupling of small- and large-scale phenomena.

Figure 9 shows the evolution of the global coronal structure above the limb in the SOHO/SDO 195/193 A images (see, e.g., Figure 7 of McIntosh et al. 2013). These images represent the temporal progression of the EUV emission integrated in a narrow $\left(1.15-1.25 R_{\odot}\right)$ annulus around the solar limb. In addition to showing the latitudinal progression of the activity bands toward the equator (the dashed lines again trace out the progression of the activity bands and are taken from Figure 6), we see that the extent of the polar coronal hole very rarely migrates closer to the equator than $\pm 55^{\circ}$. Indeed, the polar coronal holes seem to be strongly bounded by $\pm 55^{\circ}$. The main poleward excursions observed are the notable poleward surges of coronal emission and polar crown filaments-the so-called "Rush to the Poles" (e.g., Wilson et al. 1988; Altrock et al. 2008; Tappin \& Altrock 2013) —near the sunspot maxima of cycle 23 (and 24 currently ongoing).

We note that the duration of the surges appears to be identical to the length of time spent by the g-node activity bands at $\pm 55^{\circ}$ before their migration toward the equator. The g-node progression in each hemisphere and the dashed activity bands are shown above and below the central coronal plots to emphasize this correspondence. We see that the surges in the northern and southern hemisphere appear to start at the same time (in 1998) and end when the g-node bands begin their equatorward march, which correspond to the clear offset between migration start in the north (2000) and south (2002), as we have noted previously. The apparent correspondence between the duration of the polar surges and the length of time spent at $\pm 55^{\circ}$ by the (g-node) activity bands would appear to support our earlier assertion that the magnetic flux emerging from the activity band at high-latitude "feeds" the polar region with oppositely signed magnetic flux. This flux eventually closes the polar coronal hole and reverses the net flux of the polar regions after that flux is caught in the surface (poleward) meridional flow. Indeed, as we have noted, this pattern is currently repeating, indicating that the activity band representing the magnetic activity bands responsible for solar cycle 25 are present at high latitudes with surges starting in 2010 and 2012 in the northern and southern hemispheres, respectively.

Figure 10 shows the evolution of coronal holes automatically detected using EUV images and magnetograms from $\mathrm{SOHO}$ and $S D O$ (Krista \& Gallagher 2009), and places them in context with the evolution of the activity bands, the hSSN, and the underlying photospheric magnetism. The panels of the figure show the hSSN (north: red; south: blue, from Figure 6) and coronal hole characteristics for comparison with the MDI/HMI latitude-time plot, and the g-node density variation in latitude and time. In each of the lower panels, we show the horizontal dashed lines at $\pm 55^{\circ}$ and the progression of the activity bands with time (positive: red; negative: blue) to illustrate the strong correspondence between the progression of the activity bands and the identified coronal holes. ${ }^{10}$

\footnotetext{
10 A broader discussion of the coronal hole detection algorithm and the detailed study of this progression is presented in a subsequent paper (L. D. Krista et al. 2014, in preparation).
} 

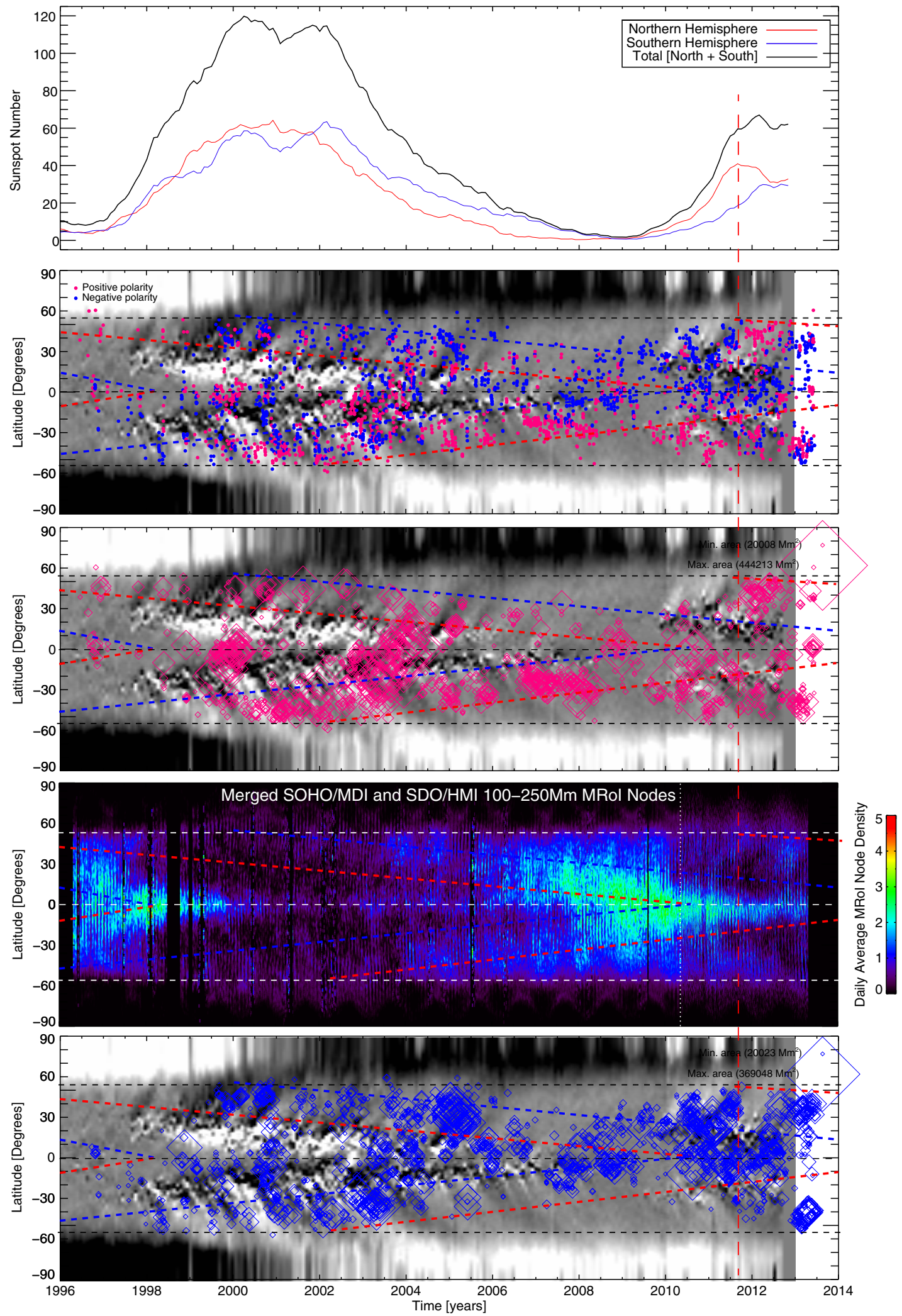

Figure 10. Results of automated coronal hole detection by the CHARM algorithm using images and magnetograms from $S O H O$ and $S D O$ (Krista \& Gallagher 2009 , and L. D. Krista et al. 2013, in preparation). From top to bottom, the panels of the figure show the hSSN (north: red; south: blue, from Figure 6), the centers of the coronal holes identified which have an area greater than $20,000 \mathrm{Mm}^{2}$ (positive—red; negative—blue) overlaid on the MDI/HMI latitude-time plot, the area (indicated by size of the diamond) and location of only those positive polarity coronal holes greater than $20,000 \mathrm{Mm}^{2}$ in area, the g-node density variation in latitude and time (see Figure 6), and the area (indicated by size of the diamond) and location of the negative and positive polarity coronal holes greater than $20,000 \mathrm{Mm}^{2}$ in area. In the lower four panels, the horizontal dashed black lines are drawn at $\pm 55^{\circ}$ and the activity bands are colored red and blue to indicate their net polarity. The vertical dashed red line running through the entire plot indicates the time of northern hemisphere solar maximum; when this paper was written, the southern hemisphere had not yet reached maximum and so there is no equivalent line (see text).

(A color version of this figure is available in the online journal.) 


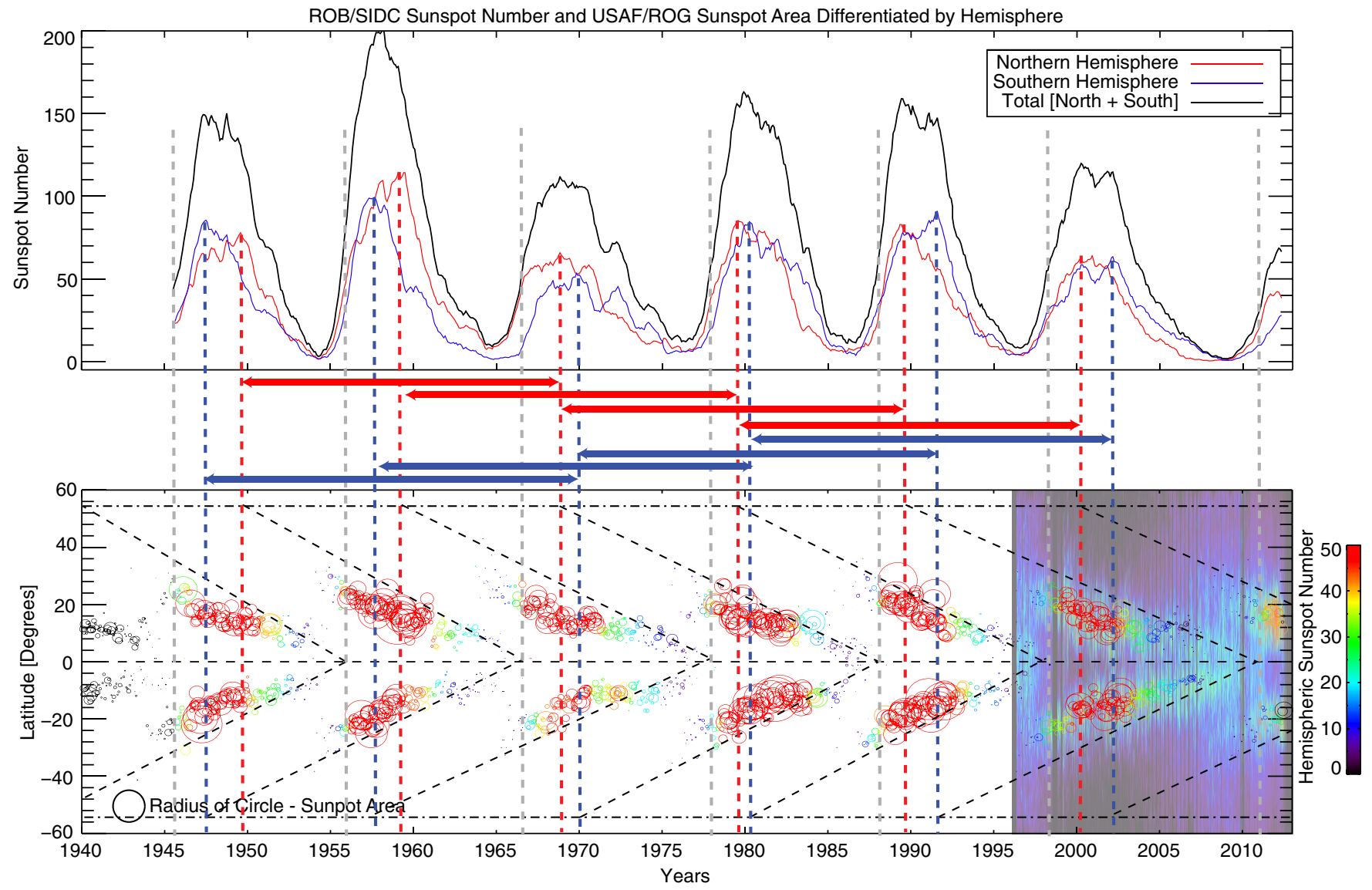

Figure 11. We extend the cycle 23 metrics to a longer epoch covering six complete sunspot cycles (1940-2012). We use a combination of the hSSN and the USAF/Royal Observatory Greenwich record of sunspot areas. The dashed lines are drawn from the hSSN maxima (red: north; blue: south) and signify the beginning of the high-latitude band formation and migration toward the equator from $\pm 55^{\circ}$. The gray dashed lines are drawn when the sunspot areas exceed 100 millionths, indicating that the equatorial bands have canceled, permitting rapid high-latitude sunspot onset. The span from the colored dashed lines at $\pm 55^{\circ}$ to the gray dashed lines at the equator defines the chevron outlining the activity cycle. For reference, the BP latitude-time image (Figure 6(c)) is shown for cycle 23. We compare this with the zonal flow over four solar cycles (Figure 12) and for the longer record of sunspot areas (Figure 13).

(A color version of this figure is available in the online journal.)

We plot the central location and area of the coronal holes with areas greater than $20,000 \mathrm{Mm}^{2}$ (chosen to represent the area covered by $\mathrm{g}$ nodes with a separation of $80 \mathrm{Mm}$ ) formed below $70^{\circ}$ latitude and color-coded by their underlying polarity (positive-red; negative-blue). We see that there is a general migration of the low-latitude coronal holes toward the equator in the band of similar polarity which links their evolution to the progression of BPs and g nodes. This progression indicates that a significant population of coronal holes have deep magnetic roots and are not necessarily the result of active region diffusion by surface flows (a subject covered in L. D. Krista et al. 2014, in preparation). Similarly, we can infer from this complex "double-helix" pattern of coronal hole locations that coronal holes will sit at $\pm 55^{\circ}$ for prolonged periods of time as a tracer of the underlying toroidal flux band prior to equatorward migration. Such coronal holes were readily visible in the southern hemisphere from 2000 to 2002. Indeed, such coronal holes are also visible in contemporary coronal imaging (and Figure 4) - possibly marking the start of the cycle 25 positive activity band at $+55^{\circ}$ (see also Section 7), which should start its progression equatorward at the northern activity maximum, shown by the red dashed vertical line. We note that the band in the southern hemisphere (negative at $-55^{\circ}$ ) may be starting to show, but we will have to wait and see if the southern activity maximum has been reached.

\section{APPLYING CYCLE 23 "RULES" TO A LONGER TIME FRAME}

Using the simple landmarks determined from the observations of cycle 23 above, we exploit the Royal Observatory of Belgium and Royal Observatory Greenwich records of sunspot number (SSN) and area, the results of which are shown in Figure 11. The dashed chevrons start their equatorward motion from $\pm 55^{\circ}$ at times determined using the hemispheric (hSSN) maxima marked by red (north) and blue (south) dashed vertical lines. From Figure 7, we determine that the low-latitude chevrons terminate when the measured sunspot areas exceed 100 millionths, which we draw as the gray dashed vertical lines.

These simply defined activity band chevrons enclose almost every sunspot in the record (see also Figures 12 and 13). Chevrons of the same sign start very close to $22 \mathrm{yr}$ apart (thick colored arrows that are colored to indicate hemisphere). To illustrate the correspondence between these figures and Figure 6, we show the BP density record (Figure 11) and the zonal flow patterns inferred from Doppler measurements of the solar photosphere taken at the $150 \mathrm{ft}$ Tower at the Mount Wilson Solar Observatory (Ulrich 2010). In the latter example, we see the clear relationship between the flow patterns and the high-lowlatitude evolution characteristic of the torsional oscillation, of which this is an analog, over the past three decades. 


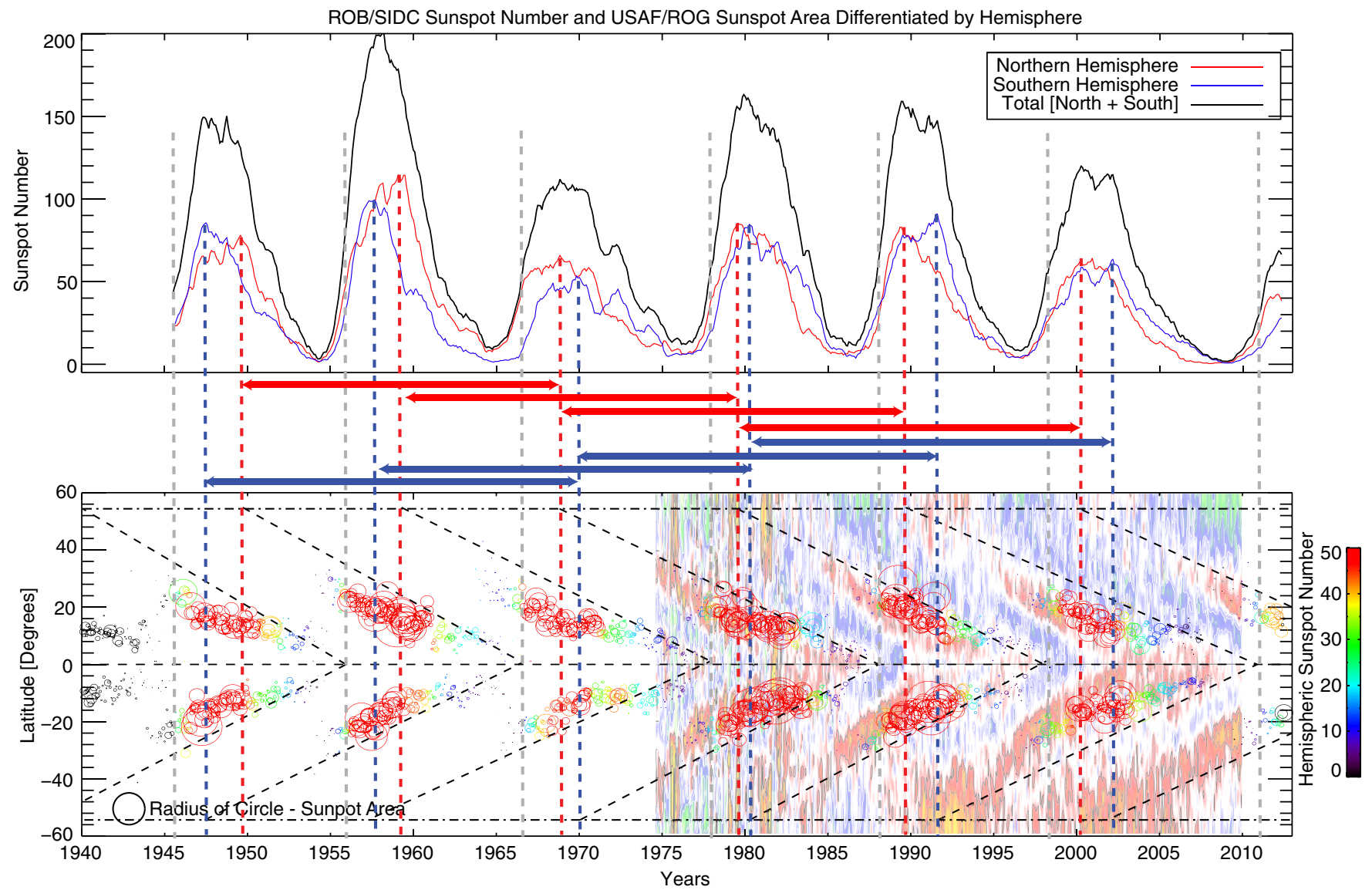

Figure 12. Comparison with Figure 11. In this instance, we have replaced the BP latitude-time distribution with the zonal flow-corresponding to the torsional oscillation (Figure 6(d); Ulrich 2010) - constructed from Doppler measurements of the solar photosphere taken at the $150 \mathrm{ft}$ Tower at the Mount Wilson Solar Observatory. Note the strong correspondence of the patterns observed with the chevrons drawn using only the hemispheric sunspot number (hSSN) as a guide. Also, note the apparent regularity of the high-latitude starting points of the consecutive chevrons.

(A color version of this figure is available in the online journal.)

Table 1

Times of Consecutive (Same-signed) Maxima, Their Difference ( $\delta$; yr), and High-Low-latitude Transit Time $(\tau ;$ yr) for Each Hemisphere Determined from Figure 13

\begin{tabular}{lcccccc}
\hline \hline Cycle Pair & Maxima $(N)$ & Maxima $(S)$ & $\delta_{N}$ & $\delta_{S}$ & $\tau_{N}$ & $\tau_{S}$ \\
\hline $14-12$ & $1905.75,1884.00$ & $1907.08,1883.83$ & 21.75 & 23.25 & 19.25 & 17.92 \\
$15-13$ & $1917.58,1892.50$ & $1919.50,1893.58$ & 25.08 & 25.92 & 19.92 & 18.00 \\
$16-14$ & $1925.92,1905.75$ & $1926.08,1907.08$ & 20.17 & 19.00 & 19.18 & 19.02 \\
$17-15$ & $1937.50,1917.58$ & $1939.67,1919.50$ & 19.92 & 20.17 & 20.50 & 18.33 \\
$18-16$ & $1949.17,1925.92$ & $1947.17,1926.08$ & 23.25 & 21.08 & 19.03 & 21.03 \\
$19-17$ & $1959.08,1937.50$ & $1956.83,1939.67$ & 21.58 & 17.17 & 19.42 & 21.67 \\
$20-18$ & $1968.00,1949.17$ & $1970.08,1947.17$ & 18.83 & 22.92 & 19.50 & 17.42 \\
$21-19$ & $1979.67,1959.08$ & $1980.33,1956.83$ & 20.58 & 23.50 & 19.33 & 18.67 \\
$22-20$ & $1989.08,1968.00$ & $1991.08,1970.08$ & 21.08 & 21.00 & 21.92 & 19.92 \\
$23-21$ & $2000.50,1979.67$ & $2002.58,1980.33$ & 20.83 & 22.25 & $\cdots$ & $\cdots$ \\
\hline
\end{tabular}

Using only the sunspot area record and the hemispheric maxima detected therein, we can compare consecutive maxima of the same sign somewhat quantitatively in Table 1 and Figure 14 where the fits determine mean evolutionary times of $20.38 \pm 1.06$ (north) and $21.63 \pm 1.52$ (south) yr (and $20.84 \pm 1.56 \mathrm{yr}$ for the average). Also from the table, we see that the equatorial bands are not regular in their timing. The transit time of the activity bands from high to low latitude appears to vary inversely with the strength of the cycle-magnetically stronger cycles have fast transits while weaker cycles take longer, implying that the circulatory speed of cells in the (coupled) system responsible for the activity cycle are critically dependent on the amount of magnetic flux that is loaded onto them.

\subsection{The NGDC Coronal Green Line Record}

The most striking feature in Figure 9 is the dearth of coronal emission above $55^{\circ}$ for the majority of solar cycle 23 . Indeed, that latitude appears to be a relatively rigid upper bound for low-latitude coronal emission, and hence a lower bound for the polar coronal hole in addition to being an upper bound for low-latitude holes (Figure 10). The only time when there is significant coronal emission at high latitudes is during the 


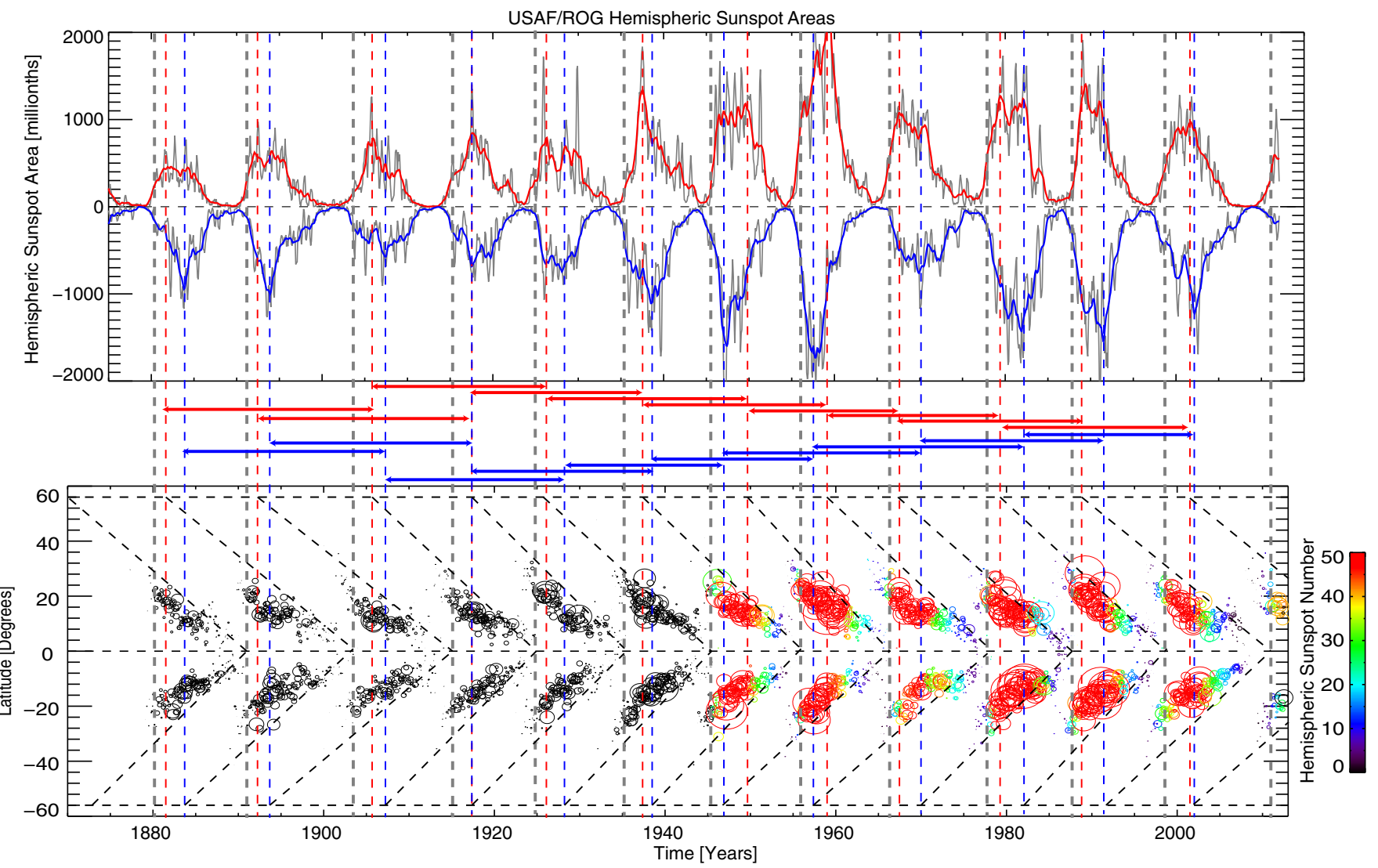

Figure 13. We use the USAF/Royal Observatory Greenwich sunspot area distributions as a proxy of hemispheric activity to extend Figures 12 and 13 into the nineteenth century. As above, the radii of the circles drawn in the lower panel indicate the percentage of the solar disk covered by sunspots (in millionths). Where we have sunspot numbers differentiated by hemisphere (after 1945), we color the circles by their hSSN. In this case, we use the hemispheric peak (red: north; blue: south) in sunspot area to identify the activity maximum in each hemisphere from which the activity band starts at $\pm 55^{\circ}$ latitude and draw the red and blue vertical dashed lines accordingly. In this case, the gray vertical lines are drawn when the sunspots exceed 100 millionths, to indicate that the equatorial bands have canceled/terminated. Again, note the strongly consistent $22 \mathrm{yr}$ repetition of the high-latitude start of the chevrons in each hemisphere.

(A color version of this figure is available in the online journal.)

progression of oppositely signed magnetic flux which reverses the polarity of the polar region. We also noted above that the duration of the coronal emission surge toward the poles in each hemisphere appears to be consistent with the length of time that the activity band (visualized in the g-node bands in the upper and lower panels) spends at $55^{\circ}$ in that hemisphere before migrating to the equator. To illustrate that these properties of coronal emission with latitude and time are not limited to sunspot cycle 23, consider Figure 15 which shows the NOAA National Geophysical Data Center (NGDC) coronal green line scan archive going back to 1939. Again, we see that the bright (hot) coronal emission is largely restricted to latitudes below $55^{\circ}$ with significant excursions poleward only taking place once every $\sim 11 \mathrm{yr}$. This record provides further support to the notion that $55^{\circ}$ is a critical latitude in solar cycle evolution. Indeed, with further work it is possible that observational records like the NGDC green line scan may be used to provide valuable information about the length of time spent by the high-latitude bands prior to their migrating equatorward.

\section{DISCUSSION}

The lengthscale on which BPs form is consistent with that of giant cell convection. We interpret the g nodes around which those BPs tend to occur (McIntosh et al. 2014) as the radial component of the deep-seated toroidal magnetic flux band rooted at (or near) the tachocline. Additional evidence in support of this interpretation can be found in the rotation rates inferred from the tracking of BPs (e.g., Golub \& Vaiana 1978) and will be explored with contemporary data in a subsequent publication (in preparation).

Giant convective cells are aligned with the Sun's rotation axis. Furthermore, the primary driver of the giant convective scale is the rotation of the radiative interior, which has a mean depth of $\sim 0.72 R_{\odot}$ (e.g., Miesch 2005, and Figure 16). However, there is strong observational evidence that the tachocline is prolate and would be shallower at polar latitudes (Charbonneau et al. 1999a). Considering the range of depths that are thus consistent with the observations would make a plane tangent to the polar tachocline cut through the Sun's surface at latitudes of $55^{\circ}-60^{\circ}$ (see McIntosh et al. 2014). In general, BPs (and the magnetic elements associated with the g nodes) are overlooked in standard latitude-time diagrams due to their relatively small spatial dimensions. Only when such features are tracked does their progression become an indicator of coherent magnetic flux emergence preceding sunspot formation (Wilson et al. 1988).

While numerical modeling of this system is absolutely required, we speculate that the deep-rooted magnetic flux bands have a simple (and continuous) ability to breach the photosphere (e.g., Weber et al. 2013). The persistent eruption of flux connected to the activity band driven by the large-scale convection above (e.g., Nelson et al. 2011, 2013, 2014) permits 


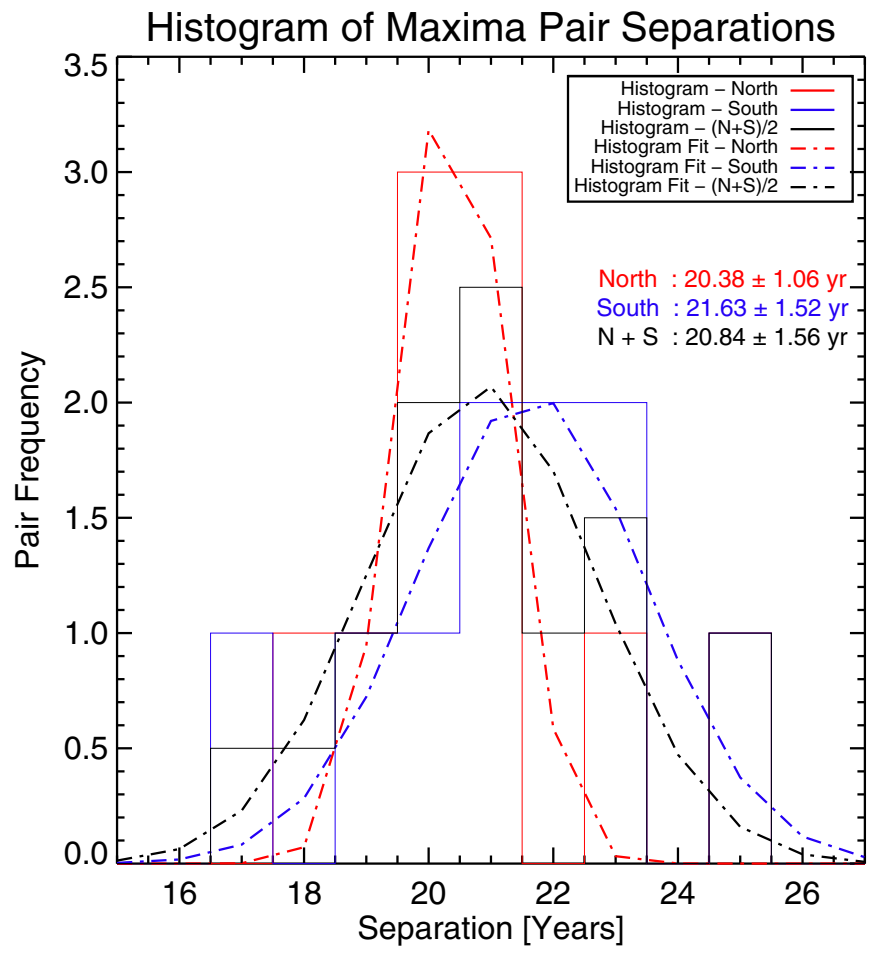

Figure 14. Histograms of same-sign solar maxima separation from the hemispheric (north-red; south-blue) maxima of the USAF/Royal Observatory Greenwich sunspot area distributions shown in Figure 13. The Gaussian fit to each distribution (dot-dashed lines) allows us to estimate the mean cycle time of the northern and southern hemispheres.

(A color version of this figure is available in the online journal.)

the progression of the flux band from birth to termination to be captured in the surface velocity field, g-node pattern, and associated BPs. The technique of mapping the progression of the underlying magnetic flux band presented in this paper has a distinct advantage over global helioseismology methods for inferring the progression of the circulatory pattern during times of profound hemispheric asymmetry.

We have also inferred that the region in latitudes higher than $55^{\circ}$ is important for the progression of the solar cycle. From the approximately constant mean overturn time of the polar cells, we propose that they act as clocks for the coupled system. The perturbations to the flow induced by the magnetic field at these high latitudes are small because the average fields themselves are small ( 1 G; Babcock \& Livingston 1958; Svalgaard \& Kamide 2013) when compared to that of the equatorial region. Indeed, this evolution of the polar cells may possibly help us to understand the long-established observational relationship between the polar field strength and the strength of the following sunspot cycle (Hathaway 2010)_if less flux erupts, then less is caught in the polar cell and so less is available to form the flux band of the cycle $22 \mathrm{yr}$ later, and so on. Furthermore, when less flux is available at high latitudes, the equatorial chevrons migrate slower toward the equator and this process appears to feed back on itself, although in this picture there is little effect on the interwoven cycle which may be weaker or stronger. This apparent alternation in the strength of the cycles could provide some clarification concerning another observational phenomenon, the Gnevyshev-Öhl or "odd-even" alternating progression of SSNs from cycle to cycle (Gnevyshev \& Öhl 1948).

The maps of torsional oscillation (Figure 6(d), or the zonal flow in Figure 16) provide a mapping of the surface magnetism discussed above. However, our article is not the first to draw this connection or to pose the following questions (Wilson et al. 1988; Altrock et al. 2008): what is the torsional oscillation and what is its connection to the $22 \mathrm{yr}$ magnetic cycle? The analysis presented appears to offer some insight into the modulation of the solar cycle. Indeed, it is remarkably consistent with the concept of ESC (Wilson et al. 1988). The ESC is an observationally derived paradigm which, over the last several decades, has persistently demonstrated an observational connection between helioseismology (Altrock et al. 2008), surface magnetic phenomena, and extended coronal features (McIntosh et al. 2013; Tappin \& Altrock 2013), which display a cyclic behavior with a period approaching the complete $22 \mathrm{yr}$ of the magnetic activity cycle.

Further work is required to understand fully the precise balance between the magnetic field and the impact on the circulatory system, how the magnetic field forms, and how it is loaded into the circulatory system. However, we can see that

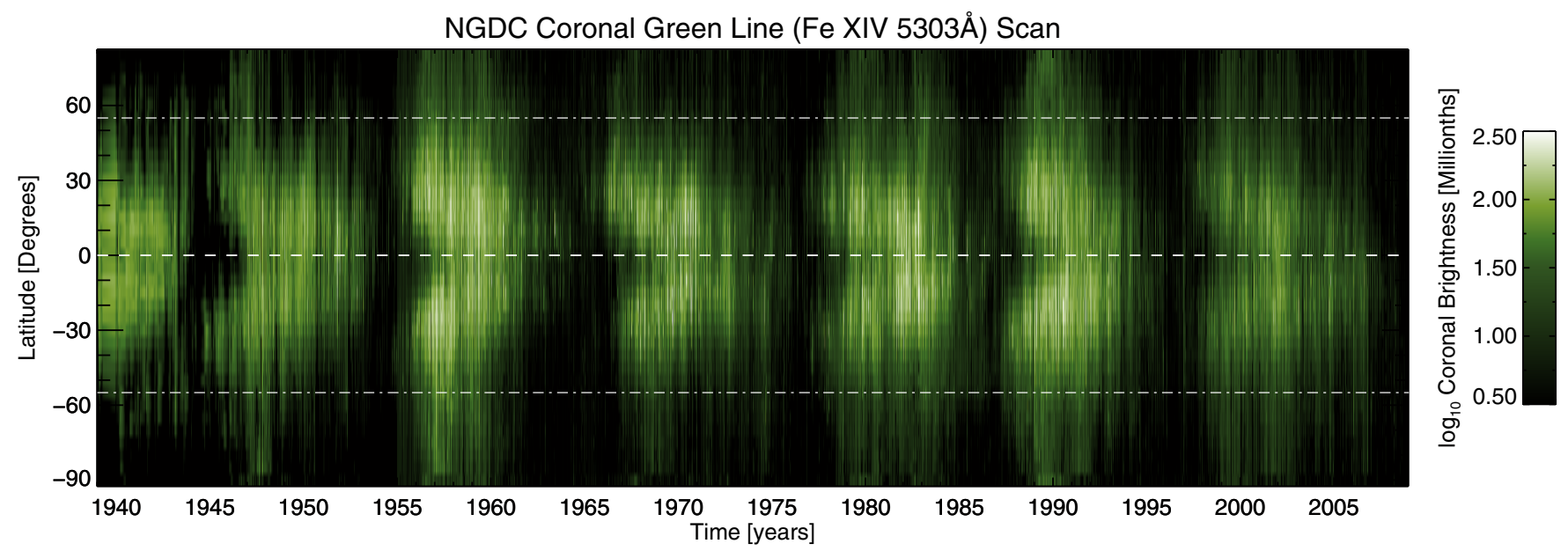

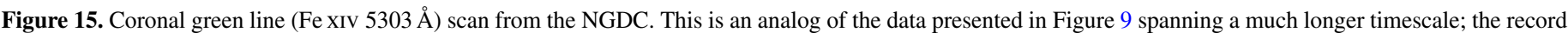

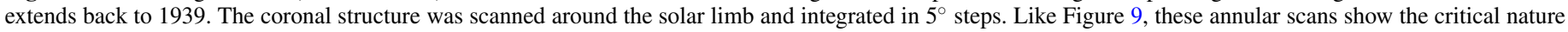

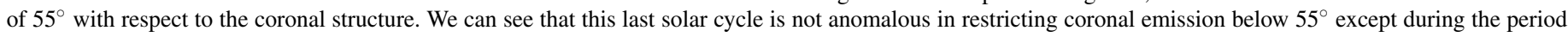
when the polar reversal is taking place. The equator is shown as a white dashed line, while the dot-dashed lines show $55^{\circ}$ in each hemisphere.

(A color version of this figure is available in the online journal.) 


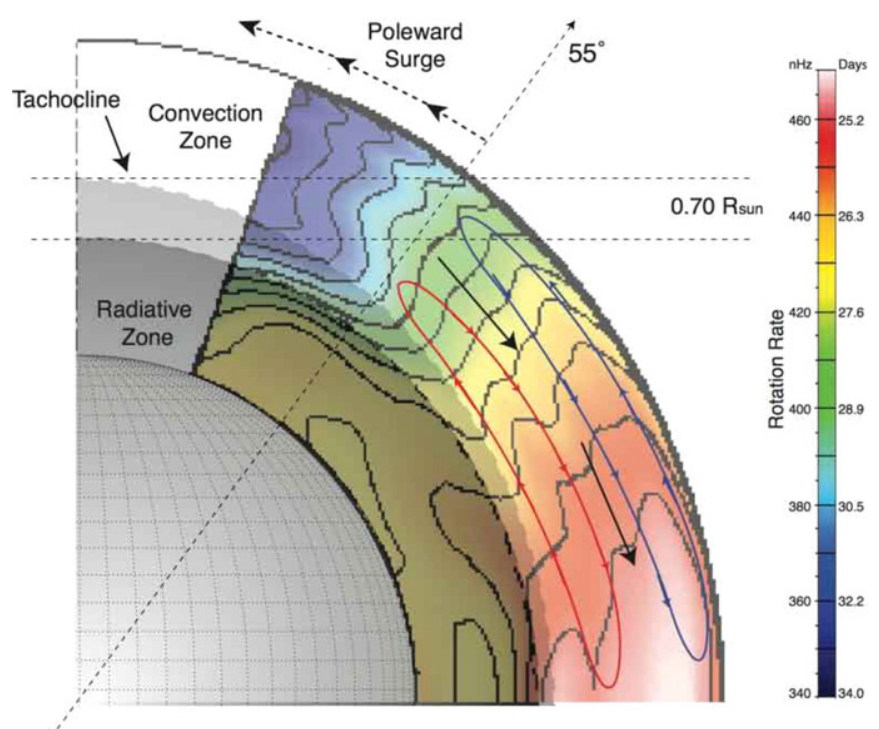

Figure 16. Cut through one quadrant of the Sun to illustrate the possible flow patterns governing the magnetic flux progression. The observations are delineated by the regions above and below $55^{\circ}$ latitude (also seen in the pattern of interior differential rotation; Thompson et al. 1996) as the projected surface latitude of the depth of the high-latitude radiative zone (darker sphere; note the prolateness of the radiative zone-see text). We also sketch the region called the tachocline (lighter sphere), one possible equatorial circulatory pattern below $55^{\circ}$ (Zhao et al. 2013) where we only have a sense of the deep progression of the magnetic elements, and a polar circulation cell above $55^{\circ}$ where we only have a sense of their surface progression.

(A color version of this figure is available in the online journal.)

stronger cycles are faster and the weaker cycles are slower, and that the strength of the cycle is dependent on the amount of flux advected into the polar cell in the preceding cycle of the same sign. There appears to be a hysteresis in the feedback between the flow and the magnetic field of the equatorial region which can establish the rising or falling strength of magnetic activity over longer timescales. Indeed, further work is also required to combine these $\mathrm{SOHO}$ and $\mathrm{SDO}$ observations with those from Kitt-Peak, Mount Wilson, and older space records of smallscale magnetic evolution to consistently probe multiple activity cycles.

The results presented above have a significant impact on many of the solar dynamo models currently employed in the community (e.g., Babcock \& Babcock 1955; Leighton 1969; Dikpati \& Charbonneau 1999; Nandy et al. 2011). In general, these models require the formation of a new poloidal flux system at the end of every $11 \mathrm{yr}$ cycle which can seed the toroidal field for the next $11 \mathrm{yr}$. However, the observations provided above would appear to demonstrate that these activity bands form at very high latitudes and are overlapping temporally and interacting with each other in the solar interior for more than half of the $22 \mathrm{yr}$ period. Therefore, we must explore new ways to explain the complexity of this coupled feedback system. The next generation of self-consistent magnetohydrodynamic (MHD) models of the solar interior may offer significant clues (Charbonneau 2010; Racine et al. 2011), as they seem to reproduce a broader range of the observed phenomena. However, if our determination that the regularity of highlatitude evolution/circulation is critical to the modulation of the coupled rotating (magneto-)hydrodynamic system, then detailed simulations and careful observations of the general circulation (and especially the polar regions) should reveal the controlling physical processes responsible for the production of the magnetic field and its evolution.

Motivated by our observational analysis, we propose that it is the polar regions, rather than the lower latitudes, which regulate the timescale of the solar activity cycle while the lower latitudes respond. The zonal flow oscillation at high latitudes may set this timescale through hydrodynamic means such as coupling to the radiative interior, or it may arise through the oscillating fields of a cyclic dynamo operating in the polar regions. Such a "polar dynamo" would account for the distinct evolution of the magnetic signatures above and below $\pm 55^{\circ}$, which is evident in many of the figures presented herein. The existence of a polar dynamo is not so fanciful as one might initially surmise. Some global MHD simulations of solar convection do sometimes produce strong fields at high latitudes, in addition to strong low-latitude toroidal flux concentrations (see, e.g., Figure 6 of Käpylä et al. 2013 and Figure 5 of Brown et al. 2011). One may also draw parallels between the "toroidal wreaths" of Brown et al. (2011) and our Figure 8. Moreover, even simple kinematic mean-field dynamos operating with a solar-like differential rotation profile tend to produce significant magnetic fields at high latitudes, unless the $\alpha$ effect is artificially concentrated at low latitudes (Charbonneau 2010). We suspect that the community's current efforts are limited to focusing on low latitudes by self-selection: either because of where visible sunspots occur, or because of the (fear of) data uncertainties from projection effects of lineof-sight magnetograms. Definitive proof must wait until highlatitude magnetogram observations are made available (e.g., the later stages of the Solar Orbiter mission when it gets above, say, $\pm 25^{\circ}$ ).

If distinct dynamos do indeed operate at the north and south poles, injecting magnetic flux into lower latitudes at different times, then what gives rise to the remarkable synchronization of the lower-latitude activity branches such that the north and south branches of the chevrons terminate at the same point? One possibility might be a nonlinear relaxation of the magnetic field roughly analogous to the "annealing" of poloidal flux discussed by Spruit (2011). If the polar dynamos inject helical flux systems into lower latitudes as illustrated in Figures 8 and 16, then the merging and cancelation of oppositely signed toroidal bands in the north and south would produce a coalescence of poloidal fields which may help to synchronize the hemispheres. Furthermore, if some of the resulting poloidal field were to thread through the photosphere, then this may account for the proliferation of BPs and g nodes near the tips of the chevrons evident in Figure 6. Alternatively, injection of flux from the polar dynamo branches may excite a symmetric dynamo wave at lower latitudes. In any case, we propose that high-latitude dynamo action acts as a "clock" to set the pace of the solar cycle.

Naturally, the "grand challenges" for any piece of research (observational or otherwise) claiming to understand the modulation of the sunspot cycle are how does the Sun enter and recover from grand minima and what can we infer about the next solar cycle (or beyond)? Furthermore, what do the intra- and extrahemispheric coupling of the activity bands at different phases of the $22 \mathrm{yr}$ period mean for flux emergence processes, flares, and coronal mass ejections? These matters will be discussed in forthcoming papers in the series.

To close on a similarly broad point, in the study of stellar activity cycles (e.g., Böhm-Vitense 2007), it is time to acknowledge that the 22 yr magnetic cycle is the Sun's "fundamental" mode 
and not $11 \mathrm{yr}$ as reflected in the pattern produced by the interaction of the activity bands. It remains to be seen how the star's rotation rate, convection zone depth, and other (relatively invariant) properties can combine to produce the spectrum of stellar activity cycles observed, but which are likely mis-characterized. For example, consider how many activity bands per hemisphere would a star that was identical in mass and convection zone depth to the Sun have if it rotated three or five times faster? The calcium index of that hypothetical star would reflect the "beat" of the overlapping bands and not the fundamental. The question must then become how do we identify the fundamental evolutionary timescale of the surface flux production without resolved observations?

\subsection{Summary of Findings}

The findings presented in this paper present a phenomenological explanation for the phases of the cyclic behavior of SSNs and appearance.

1. The $\sim 11$ yr sunspot cycle is a result of the interaction between the (temporally) overlapping toroidal activity bands of the 22 yr magnetic activity cycle. The (oppositely signed in each hemisphere) activity bands take $\sim 19 \mathrm{yr}$ from their emergence at high latitudes $\left( \pm 55^{\circ}\right)$ to reach the equator.

(a) The time between the onset of emergence of activity bands of the same sign at high latitude in the same hemisphere is $\sim 20.84( \pm 1.56) \mathrm{yr}$.

2. The magnetic activity bands are rooted in the deep interior, and small-scale magnetic flux emergence from them allows their passage to be tracked in a set of synoptic observations.

(a) The MRoI presents a possible observational link to giant cell convection through the $\sim 100 \mathrm{Mm}$ lengthscales observed.

(b) Coronal BPs form almost exclusively around the vertices of these giant convective cells, "g nodes."

(c) Identifying MRoI g nodes and BPs allows us to track the progress of the activity bands.

(d) The overlapping activity bands match the progression of the torsional oscillation, linking surface magnetism to helioseismic inference.

(e) The progression of low-latitude coronal holes also allows us to track the progress of the activity bands.

(f) There is only one surge of magnetic activity that is swept into the polar region by the surface meridional circulation. The duration of that surge appears to match the length of time spent by the activity bands at high latitudes prior to starting their migration equatorward.

3. The intra-hemispheric and extra-hemispheric communication of the activity bands modulates the appearance of sunspots (see Figure 9).

(a) The activity bands form "chevrons." The overlap in time of these chevrons, when compared directly to the variation of the hSSN, provides observational clues to understanding the phases of the solar cycle.

i. When no opposite activity band exists in the same hemisphere, sunspots can abundantly emerge rooted in the existing activity band. There is only communication across the equator between the activity bands. This defines the rapid SSN growth described as the "ascending phase." ii. The emergence of the new (oppositely signed) high-latitude activity band marks a downturn in the occurrence of sunspots in each hemisphere. The two bands mutually interfere, hence reducing the ability of the low-latitude band to produce sunspots. The point of downturn defines "solar maximum," and marks the start of the "declining phase."

iii. The activity bands are both migrating to the equator under the action of global circulation, which is (probably) a result of the Sun's rotation. The latitudinal offset between the oppositely signed activity bands may be important for (large) flare production if oppositely signed flux emergence readily takes place neighboring, or in, a preexisting active region.

iv. When the two low-latitude activity bands eventually "terminate" at the equator, sunspots appear shortly thereafter following the path of the higherlatitude bands (now at around $30^{\circ}$ latitude) within only one or two solar rotations. This marks the start of the next ascending phase.

v. This concept of overlapping, oppositely signed, activity bands significantly changes the paradigm of "solar minimum." In the context presented, solar minimum is a time of maximal overlap and cancelation between the activity bands in their own hemisphere and across the equator.

4. Based on the observations studied, the degree of overlap between the chevrons in the equatorial region of the "extended solar cycle" dictate the shape, length, and magnitude of the sunspot cycle. These factors likely impact the pattern of solar energetic output (e.g., McIntosh et al. 2013). There appears to be significant feedback between the magnetic field and the circulatory flow.

(a) More magnetic flux in the equatorial activity bands speeds up the transition from high to low latitude and gives rise to strong cycles.

(b) Less magnetic flux in the equatorial activity bands slows down the progression and lengthens the overlap of the chevrons. This increasing overlap results in a weak cycle and fewer sunspots.

(c) The latter appears to the be an ever-decreasing state-the reduction of flux in the equatorial region begets another.

(d) The transit time may be a robust indicator of the strength of the subsequent sunspot cycle.

\section{FORECASTING SUNSPOT CYCLE 25 ONSET}

The gradient of the fitted straight lines and their associated error can be used to project the migration of the activity bands to a point where they will cross (as shown in Figure 7). The crossing point indicates the time at which we expect the termination of the cycle 24 bands and the strong emergence of cycle 25 sunspots may occur. This "forecast" is made under the assumption that the activity band migration continues at the same linear rate, an assumption which will need to be relaxed in the future once the entire migratory period has been monitored. The pink dashed lines shown in Figure 17 assume that the gradient of latitudinal migration toward the equator does not vary from the fitted value while the dotted lines reflect the minimum and maximum permissible gradients to the fit within the linear assumption. 


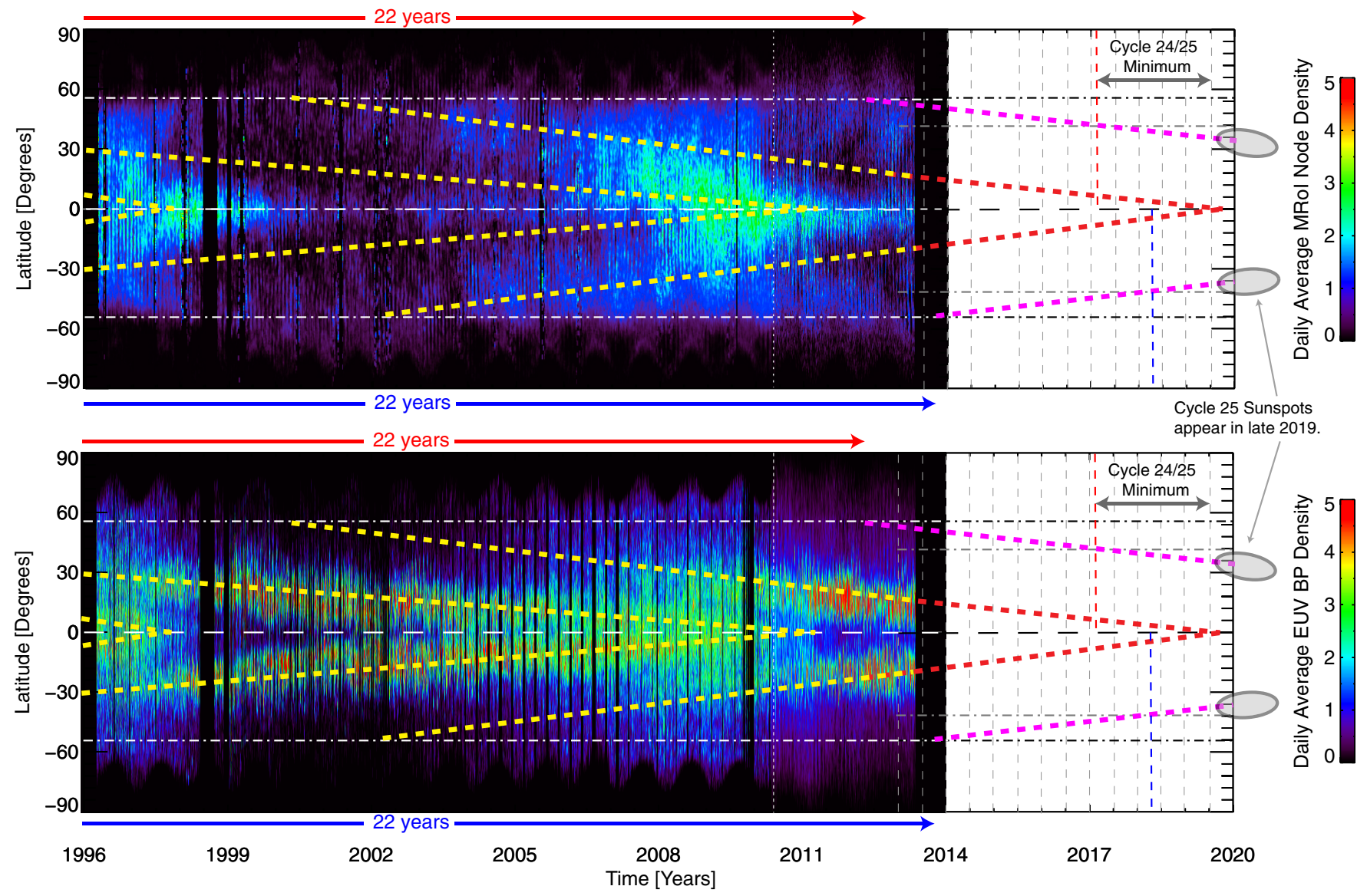

Figure 17. We use the panels of Figure 6 to extrapolate the termination of the BP chevrons based on the metrics determined above. The chevrons (yellow dashed lines) are taken from Figure 6 and the faint vertical dashed gray lines are six months apart. The red dashed lines are the linear continuation of the yellow cycle 24 bands. The cycle 25 bands appear as pink dashed lines starting at high latitudes $22 \mathrm{yr}$ from the start of their cycle 23 counterparts (derived from Figures 11 and 12). The red and blue vertical dashed lines indicate the possible onset of solar minimum conditions in the northern and southern hemispheres.

(A color version of this figure is available in the online journal.)

We see that the inner dotted lines cross in the second half of 2017, while the outer dotted lines cross in the last quarter of 2019; the region between these points has been shaded. If the current activity bands slow to the rates shown by the activity bands of cycle 22 and cycle 23 in the last few years of their migration, then the lines shown will become the earliest likely dates for cycle 24 termination and cycle 25 onset.

\section{CONCLUSION}

We conclude that observations covering the past $17 \mathrm{yr}$ with $S O H O$ and SDO indicate that the spatio-temporal evolution of the $11 \mathrm{yr}$ sunspot cycle is a result of the interaction between the temporally overlapping activity bands belonging to the 22 yr magnetic activity cycle. Synoptic monitoring of the solar photosphere and corona will continue in order to test this hypothesis and validate our forecast that the sunspots of solar cycle 25 will begin to appear as early as the end of this decade.

The data used in this paper are openly available from the $S O H O, S D O$, and the Virtual Solar Observatory (VSO; http://virtualsolar.org) data archives. S.W.M., R.J.L., A.R.D., and R.S.M. were partly funded by NASA grants (NNX08AU30G, NNX08AL23G, NNM07AA01C-Hinode, NNG09FA40C-IRIS). A.V.M. is supported by NASA grant NNG04EA00C (SDO/AIA). SOHO is a project of international collaboration between ESA and NASA. Sunspot data are from
David Hathaway and the World Data Center SILSO, Royal Observatory of Belgium, Brussels. S.W.M. is grateful to Matthias Rempel, Yuhong Fan, Dick Altrock, Sara Martin, Roger Ulrich, Paul Charbonneau, Mark Miesch, and Eugene Parker for helpful discussions. NCAR is sponsored by the National Science Foundation.

\section{REFERENCES}

Altrock, R., Howe, R., \& Ulrich, R. 2008, in ASP Conf. Ser. 383, Subsurface and Atmospheric Influences on Solar Activity, ed. R. Howe, R. W. Komm, K. S. Balasubramaniam, \& G. J. D. Petrie (San Francisco, CA: ASP), 335

Babcock, H. D. 1959, ApJ, 130, 364

Babcock, H. D., \& Livingston, W. C. 1958, Sci, 127, 1058

Babcock, H. W., \& Babcock, H. D. 1955, ApJ, 121, 349

Böhm-Vitense, E. 2007, ApJ, 657, 486

Brown, B. P., Miesch, M. S., Browning, M. K., Brun, A. S., \& Toomre, J. 2011, ApJ, 731, 69

Charbonneau, P. 2010, LRSP, 7, 3

Charbonneau, P., Christensen-Dalsgaard, J., Henning, R., et al. 1999a, ApJ, 527,445

Charbonneau, P., Dikpati, M., \& Gilman, P. A. 1999b, ApJ, 526, 523

Delaboudinière, J.-P., Artzner, G. E., Brunaud, J., et al. 1995, SoPh, 162, 291

Dikpati, M., \& Charbonneau, P. 1999, ApJ, 518, 508

Gnevyshev, M. N., \& Öhl, A. I. 1948, AZh, 25, 18

Golub, L., \& Vaiana, G. S. 1978, ApJL, 219, L55

Hale, G. E. 1924, Natur, 113, 105

Hale, G. E., Ellerman, F., Nicholson, S. B., \& Joy, A. H. 1919, ApJ, 49, 153

Hara, H., \& Nakakubo-Morimoto, K. 2003, ApJ, 589, 1062

Harvey, K. L. (ed.) 1992, in ASP Conf. Ser. 27, The Solar Cycle (San Francisco, CA: ASP), 335 
Hathaway, D. H. 1996, ApJ, 460, 1027

Hathaway, D. H. 2010, LRSP, 7, 1

Howe, R. 2009, LRSP, 6, 1

Howe, R., Christensen-Dalsgaard, J., Hill, F., et al. 2000, Sci, 287, 2456

Käpylä, P. J., Mantere, M. J., Cole, E., Warnecke, J., \& Brandenburg, A 2013, ApJ, 778, 41

Krista, L. D., \& Gallagher, P. T. 2009, SoPh, 256, 87

Labonte, B. J., \& Howard, R. 1982, SoPh, 75, 161

Leighton, R. B. 1969, ApJ, 156, 1

Lemen, J. R., Title, A. M., Akin, D. J., et al. 2012, SoPh, 275, 17

Maunder, E. W. 1904, MNRAS, 64, 747

McIntosh, S. W. 2007, ApJ, 670, 1401

McIntosh, S. W., Davey, A. R., \& Hassler, D. M. 2006, ApJL, 644, L87

McIntosh, S. W., Davey, A. R., Hassler, D. M., et al. 2007, ApJ, 654, 650

McIntosh, S. W., \& Gurman, J. B. 2005, SoPh, 228, 285

McIntosh, S. W., Leamon, R. J., \& De Pontieu, B. 2010, ApJ, 727, 7

McIntosh, S. W., Leamon, R. J., Gurman, J. B., et al. 2013, ApJ, 765, 146

McIntosh, S. W., Wang, X, Leamon, R. J., \& Scherrer, P. H. 2014, ApJL, 748,32

Miesch, M. S. 2005, LRSP, 2, 1

Nandy, D., Muñoz-Jaramillo, A., \& Martens, P. C. H. 2011, Natur, 471, 80

Nelson, N. J., Brown, B. P., Brun, A. S., Miesch, M. S., \& Toomre, J. 2011, ApJL, 739, L38
Nelson, N. J., Brown, B. P., Brun, A. S., Miesch, M. S., \& Toomre, J. 2013, ApJ, 762,73

Nelson, N. J., Brown, B. P., Sacha Brun, A., Miesch, M. S., \& Toomre, J. 2014, SoPh, 289, 441

Racine, É., Charbonneau, P., Ghizaru, M., Bouchat, A., \& Smolarkiewicz, P. K. 2011, ApJ, 735, 46

Scherrer, P. H., Bogart, R. S., Bush, R. I., et al. 1995, SoPh, 162, 129

Scherrer, P. H., Schou, J., Bush, R. I., et al. 2012, SoPh, 275, 207

Schwabe, M. 1844, AN, 21, 233

Sheeley, N. R., Jr. 2005, LRSP, 2, 5

Spruit, H. C. 2011, in The Sun, the Solar Wind, and the Heliosphere, ed. M. P. Miralles \& J. Snchez Almeida (IAGA Special Sopron Book Series, Vol. 4; Berlin: Springer), 39

Svalgaard, L., \& Kamide, Y. 2013, ApJ, 763, 23

Tappin, S. J., \& Altrock, R. C. 2013, SoPh, 282, 249

Thompson, M. J., Toomre, J., Anderson, E. R., et al. 1996, Sci, 272, 1300

Ulrich, R. K. 2010, ApJ, 725, 658

Vaiana, G. S., Krieger, A. S., \& Timothy, A. F. 1973, SoPh, 32, 81

Weber, M. A., Fan, Y., \& Miesch, M. S. 2013, SoPh, 287, 239

Wilson, P. R., Altrock, R. C., Harvey, K. L., Martin, S. F., \& Snodgrass, H. B. 1988, Natur, 333, 748

Zhao, J., Bogart, R. S., Kosovichev, A. G., Duvall, T. L., \& Hartlep, T., Jr. 2013, ApJL, 774, L29 